\title{
Associations of short-term exposure to air pollution with respiratory hospital admissions in Arak, Iran
}

\author{
Mostafa Vahedian ${ }^{1}$, Narges Khanjani ${ }^{2,5^{*}}$, Moghaddameh Mirzaee $^{3}$ and Ali Koolivand ${ }^{4}$
}

\begin{abstract}
Background: Ambient air pollution, is one of the most frequently stated environmental problems. Many epidemiological studies have documented adverse health effects for ambient air pollution. This study aimed to investigate the association between ambient air pollution and respiratory hospital admissions.

Methods: In this ecological time series study data about air pollutant concentrations including $\mathrm{CO}, \mathrm{NO}_{2}, \mathrm{O}_{3}, \mathrm{PM}_{2.5}$, $\mathrm{PM}_{10}$ and $\mathrm{SO}_{2}$ and, respiratory hospital admissions in the urban population of Arak, from January 1 st 2010 to December 31st 2015; were inquired, from the Arak Department of Environment, and two major hospitals, respectively. Meteorological data were inquired for the same period as well. Time-series regression analysis with a distributed lag model, controlled for seasonality long-time trends, weather and day of the week, was used for data analysis.

Results: Every $10 \mu \mathrm{g} / \mathrm{m}^{3}$ increase in $\mathrm{NO}_{2}$, and $\mathrm{PM}_{10}$ and every $1 \mathrm{mg} / \mathrm{m}^{3}$ increase in $\mathrm{CO}$ at lag 0 corresponded to a RR $=1.032(95 \% \mathrm{Cl}, 1.003-1.06), \mathrm{RR}=1.01(95 \% \mathrm{Cl}, 1.004-1.017)$ and $\mathrm{RR}=1.09(95 \% \mathrm{Cl}, 1.04-1.14)$, increase in respiratory disease hospitalizations, respectively. Males and the elderly were found to be more susceptible than females and other age groups to air pollutants in regard to respiratory disease admissions.
\end{abstract}

Conclusions: The results of this study showed that outdoor air pollutants significantly increase respiratory hospital admissions; especially among the men and elders in Arak.

Keywords: Air pollution, Respiratory hospital admissions, Time-series regression

\section{Background}

Ambient air pollution, which has been exacerbating over the last few decades in the world, is one of the most frequently stated environmental problems, especially in the developing countries [1-4]. This global public health concern was estimated to cause 3.7 million rural and urban premature deaths worldwide in 2012 [5]. Many epidemiological studies have documented increase in outdoor air pollution concentrations associated with adverse health effects, including increased respiratory hospital admission [4, 6-11].

Previous published studies in the world have shown that air pollutants are related to cardiac and respiratory

\footnotetext{
* Correspondence: n_khanjani@kmu.ac.ir

${ }^{2}$ Environmental Health Engineering Research Center, Kerman University of

Medical Sciences, Kerman, Iran

${ }^{5}$ Department of Epidemiology and Biostatistics, School of Public Health,

Kerman University of Medical Sciences, Kerman, Iran

Full list of author information is available at the end of the article
}

deaths and hospital admissions [12-15]. The adverse effects of increasing air pollution, indicated as an increase in respiratory hospital admissions has been reported from North America and Europe [13-19], and relatively fewer studies of this kind have been conducted in developing countries and the Middle East. For example, a study from Italy found a positive association for $\mathrm{PM}_{10}$, $\mathrm{SO}_{2}, \mathrm{NO}_{2}$ and $\mathrm{CO}$ with respiratory disease hospital admissions [15]. In another study in two northern New England cities, an interquartile range (IQR) increase in $\mathrm{SO}_{2}$ and $\mathrm{O}_{3}$ were associated with increase in all respiratory and asthmatic emergency room (ER) visits, in Portland. However, no significant associations between air pollution and respiratory ER visits were found in Manchester, UK [19].

Some studies conducted in Asia have also found a positive relation between air pollutants and respiratory hospital admission [20-24]. For example, one study in 
Beijing, showed that an increase in $\mathrm{NO}_{2}, \mathrm{PM}_{10}$, and $\mathrm{SO}_{2}$ were associated with an increase in respiratory disease emergency admissions [1]. Another study in Tehran, Iran found that an increase in $\mathrm{PM}_{2.5}, \mathrm{NO}_{2}, \mathrm{CO}$, and $\mathrm{O}_{3}$, were respectively associated with an increase in respiratory hospital admissions [23].

However, the findings of developed countries might not be generalizable to developing countries because of the different constituents of ambient air pollution or the different demographics in their communities [25]. In developing countries, air pollution is increasingly becoming a major healthcare issue because of increased motor vehicles, traffic, lack of appropriate control on pollutant resources, industrialization [26] and lack of proper legislations. In these countries air pollution has had an ascending trend [26, 27].

Iran, is a developing country, [27], experiencing demographic and epidemiological transition and environmental pollution issues. These changes are due to its accelerated urbanization and industrialization [28], and increasing vehicles which have led to heavy traffic in cities [29], which is leading to increased levels of air pollutants. Arak is an industrialized city in central Iran and is one of the most polluted cities in the country, due to its heavy industrial activities, high number of motor vehicles, traffic and growing population.

Previous studies have reported associations between short-term air pollution exposure and respiratory deaths and hospitalization $[13,15,17,19,20]$. Most of these studies were conducted in developed countries, but, because of the susceptibility of different populations, different levels of ambient air pollutants, and characteristics of specific air pollutants, there is still a need to investigate the health effects of ambient air pollution exposure on human health in developing countries including Iran which there are few studies about the health impact of air pollutants especially in industrial cities such as Arak. This study aimed to investigate the short-term association between daily exposures to ambient air pollutants $\left(\mathrm{NO}_{2}, \mathrm{CO}, \mathrm{PM}_{2.5}, \mathrm{PM}_{10}, \mathrm{SO}_{2}\right.$ and $\left.\mathrm{O}_{3}\right)$ and respiratory hospital admissions in the urban population of Arak in a 6-year period.

\section{Methods}

\section{Study area}

This study was conducted in Arak, Iran which is the capital of the Markazi Province. Arak has a population of about 600,000 and includes 6 urban districts. It is located in the center of Iran and is about $288 \mathrm{~km}$ from Tehran. The total area of this city is $7178.98 \mathrm{~km}^{2}$ and it stands $1748 \mathrm{~m}$ above sea level. The weather of this city is relatively warm and dry in summer, and cold and humid in winter [30]. Its maximum temperature may raise up to $35{ }^{\circ} \mathrm{C}$ in summer and fall to below $-15{ }^{\circ} \mathrm{C}$ in winter. The average annual rainfall is around $350 \mathrm{~mm}$ and the relative humidity is $46 \%$. The annual average temperature is $13.9{ }^{\circ} \mathrm{C}$. The geographic coordinates of this city are $34^{\circ} 5^{\prime} 30^{\prime \prime} \mathrm{N}$ and $49^{\circ} 41^{\prime} 21^{\prime \prime} \mathrm{E}$. Arak is an active industrial city [31], and suffers from severe air pollution in the last decades, because of the presence of different emission sources, including industrial activities, increased number of motor vehicles and population growth [31-33].

\section{Data collection}

This study was an ecological (population based) time series study. Air quality data was inquired from the archive of the Air Quality Monitoring Unit of the Arak Department of Environment from January $1^{\text {st }} 2010$ to December $31^{\text {st }}, 2015$. Data prior to this period was not included due to the high percentage of missing data. Hourly air quality data is collected routinely in Arak in 4 fixed air pollution monitoring sites, located in the urban area of Arak. These stations routinely measure 6 air pollutants including $\mathrm{CO}, \mathrm{PM}_{2.5}, \mathrm{SO}_{2}, \mathrm{NO}_{2}, \mathrm{PM}_{10}$ and $\mathrm{O}_{3}$. The location of these monitoring stations is not in the proximity of industrial polluters or major traffic sites and have sufficient distance from emitting sources.

In this study, we used the daily average concentrations of $\mathrm{CO}, \mathrm{PM}_{2.5}, \mathrm{SO}_{2}, \mathrm{NO}_{2}, \mathrm{PM}_{10}$ and $\mathrm{O}_{3}$ (maximum 8-h moving average). If the concentration of a pollutant was not available in one monitoring station on a given day, the average values from the remaining stations were used to compute the average. The percent of missing values during the study period of 2191 days was $20 \%$ for CO, $25 \%$ for $\mathrm{PM}_{10}, 30 \%$ for $\mathrm{PM}_{2.5}, 32 \%$ for $\mathrm{NO}_{2}, 34 \%$ for $\mathrm{O}_{3}$ and $35 \%$ for $\mathrm{SO}_{2}$. In this study, we imputed the missing air pollution data by using the EM algorithm method [34].

Meteorological data, including daily minimum, maximum, and average temperatures and minimum, maximum and average relative humidity, were obtained from the Arak Meteorological Organization for the same period.

Daily Hospital admissions were inquired for the same time period from two major hospitals (Amir-al-Momenin and Amir Kabir) in Arak. These two hospitals are governmental medical centers that admit people from various locations of this city. Another hospital in this city is the Qods private hospital which admits much less patients and has only 150 beds. The daily count of respiratory hospital admissions was aggregated by sex, date of hospital admission, age, and diagnosis according to the tenth revision of the International Classification of Diseases ((ICD-10) code J00-J99). The medical records information was extracted and was entered in standardized forms. We focused on the daily number of total hospital admissions occurring among the resident population in Arak city. 


\section{Data analysis}

A time-series regression analysis [35] was used to examine the short-term relationship between the count of respiratory admissions and air pollutants exposures $(\mathrm{CO}$, $\mathrm{NO}_{2}, \mathrm{O}_{3}, \mathrm{PM}_{2.5}, \mathrm{PM}_{10}$ and $\mathrm{SO}_{2}$ ). This study used Generalized Linear Models (GLM) and Distributed Lag Models (DLM) within the family of Poisson distribution [35, 36], (Eq.1). We checked Poisson regression assumptions and because of its over dispersion we use quasi-Poisson regression models. In order to estimate the association between daily air pollutants and respiratory hospital admissions, the main exposure variable was the daily level of each individual air pollutant and the dependent variables were the daily counts of respiratory hospital admissions.

The Distributed Lag Model was used for lags up to 7 days (0-7 days), in order to evaluate the delayed effect of air pollutants $[1,36]$. In order to control for seasonality and long-term trend in the data, a flexible spline function of time with 7 degrees of freedom (df) per year was used [35]. Also in order to adjust for the effects of temperature and relative humidity as potential confounders that change from day to day a natural cubic spline functions with $4 \mathrm{df}$ was used for each [35-37]. The selection of degrees of freedom was based on minimizing Akaike's Information Criterion (AIC). As almost all similar papers had used a number $<10$ for degrees of freedom, we tried 1 to 10 for the initial model and we used the number that had the lowest AIC for seasonality, long-term trend, temperature and relative humidity as the degrees of freedom (df).

Further, the day of the week (DOW) was also introduced into the model to adjust for the day of the week effect on hospital admissions. This variable shows the time interval from the previous holiday in days. Finally, we provide separate models for each pollutant, to reduce potential co-linearity between them [36]. The final model was described as below, (Eq. 1):

$$
\begin{aligned}
& \mathrm{Yt} \sim \operatorname{Poisson}(\mu \mathrm{t}) \\
& \begin{aligned}
\operatorname{Ln}(\mu t)=a+\sum_{t=0}^{7} \beta \iota A P \iota & +s(\text { time }, 7 * \text { year }) \\
& +s(T, 4 d f)+s(H, 4 d f) \\
& +\gamma D O W+E
\end{aligned}
\end{aligned}
$$

where, $t$ refers to the day of the observation, Yt is the observed daily count of respiratory hospital admissions on day $t, s$ denotes to a spline function, AP indicates the daily level of the air pollutants $\left(\mathrm{PM}_{10}, \mathrm{NO}_{2}, \mathrm{CO}, \mathrm{SO}_{2}\right.$, $\mathrm{PM}_{2.5}$ or $\left.\mathrm{O}_{3}\right), \iota$ is the lag days, $\mathrm{T}$ is the average daily temperature, $\mathrm{H}$ is the average daily relative humidity. DOW is day of the week and $E$ is error.

Lag terms were modelled separately and all together in unconstrained and constrained adjusted models. Additionally, sex (male, female) and age (under 60, and 60+ years) groups were modeled separately.

All statistical analyses were performed using $\mathrm{R}$ software version 3.3.1 (2016-06-21) ( $\mathrm{R}$ Foundation for Statistical Computing, Vienna, Austria) [38] with time-series analyses using the "dlnm" package [39]. The results were presented as the Rate Ratio (RR) and its 95\% confidence interval (CI) for daily respiratory hospital admissions, per $10 \mu \mathrm{g} / \mathrm{m}^{3}$ increase in each pollutant and per $1 \mathrm{mg}$ / $\mathrm{m}^{3}$ increase in $\mathrm{CO}$.

\section{Results}

\section{Descriptive statistics}

Summary statistics of respiratory hospital admissions, air pollutant concentrations, and meteorological data are provided in Table 1 . The total number of respiratory hospital admissions for all ages were 15,622 during the study period and the daily mean count of respiratory admissions was 7.13. More than half (57.4\%) of the respiratory hospital admissions were males, and the sex ratio was $1.35: 1$ (8966:6656) and 4843 (31\%) of the respiratory admissions were in the elderly age group (60+ year-olds).

Meanwhile, the daily average pollutant concentrations were $24.30 \mu \mathrm{g} / \mathrm{m}^{3}$ (from 0.7 to $171.21 \mu \mathrm{g} / \mathrm{m}^{3}$ ) for $\mathrm{PM}_{2.5}$ and $86.63 \mu \mathrm{g} / \mathrm{m}^{3}$ (from 2.3 to $536.28 \mu \mathrm{g} / \mathrm{m}^{3}$ ) for $\mathrm{PM}_{10}$ and these two concentrations were higher than the WHO 2014 guideline thresholds [40] which are 10 and $20 \mu \mathrm{g} / \mathrm{m}^{3}$ respectively (Table 1 ). The temporal pattern of air pollutants and daily total respiratory hospital admissions in the study period are showed in Fig. 1.

Table 2 and Fig. 2 shows the exposure-response relationship between air pollutants and respiratory hospital admissions for different lags and after adjustment for the long-term trend, weather conditions and DOW in single-pollutant models. Overall, the associations between each air pollutant and the number of respiratory hospital admissions were found to be significant for $\mathrm{NO}_{2}$ $(P=0.038), \mathrm{PM}_{10}(p=0.002)$ and $\mathrm{CO}(p=0.005)$, and the corresponding RRs and (95\% CI) were 1.032(1.0031.06), 1.01(1.004-1.017) and 1.09(1.04-1.14) per $10 \mu \mathrm{g} /$ $\mathrm{m}^{3}$ increase in the concentrations of pollutants or $1 \mathrm{mg} /$ $\mathrm{m}^{3}$ increase in $\mathrm{CO}$, at lag 0 (day). $\mathrm{O}_{3}$ showed negative association with respiratory hospital admissions $(P=$ 0.002), the corresponding RRs and $(95 \% \mathrm{CI})$ were 0.975 (95\% Cl: $0.96-0.99)$ per $10 \mu \mathrm{g} / \mathrm{m}^{3}$ increase at lag 0 day. Three air pollutants had lag effects, $\mathrm{PM}_{10}$ at lag 1 day, $\mathrm{O}_{3}$ at lag 1 day and $\mathrm{PM}_{2.5}$ at lag 7 day.

Table 3 and Fig. 3 show the exposure-response relationship between air pollutants and respiratory hospital admissions for every $10 \mu \mathrm{g} / \mathrm{m}^{3}$ increase in pollutant's concentrations and every $1 \mathrm{mg} / \mathrm{m}^{3}$ increase in CO concentrations; for different lags, in single-pollutant models among different genders. Significant effects were observed for $\mathrm{CO}$ at lag-0 day $(P=0.026)$ and lag 7 day $(P=$ 
Table 1 Descriptive Statistics of air pollution levels, meteorological variables, and hospital admissions in Arak, 2010-2015

\begin{tabular}{|c|c|c|c|c|c|c|}
\hline Variables & Mean \pm SD & Minimum & $25^{\text {th }}$ percentile & Median & $75^{\text {th }}$ percentile & Maximum \\
\hline $\mathrm{O}_{3}\left(\mu \mathrm{g} / \mathrm{m}^{3}\right)$ & $59.58 \pm 26.7$ & 1.5 & 41.47 & 55.97 & 72.82 & 186.03 \\
\hline $\mathrm{CO}\left(\mathrm{mg} / \mathrm{m}^{3}\right)$ & $2.89 \pm 0.76$ & 0.25 & 2.39 & 2.88 & 3.37 & 5.97 \\
\hline $\mathrm{SO}_{2}\left(\mu \mathrm{g} / \mathrm{m}^{3}\right)$ & $54.83 \pm 33.3$ & 1.59 & 37.49 & 47.87 & 61.91 & 566.85 \\
\hline $\mathrm{PM}_{2.5}\left(\mu \mathrm{g} / \mathrm{m}^{3}\right)$ & $24.3 \pm 20.9$ & 0.7 & 8.3 & 17.5 & 36.7 & 171.2 \\
\hline $\mathrm{PM}_{10}\left(\mu \mathrm{g} / \mathrm{m}^{3}\right)$ & $86.6 \pm 44.3$ & 2.3 & 62.1 & 82.04 & 99.3 & 536.3 \\
\hline $\mathrm{NO}_{2}\left(\mu \mathrm{g} / \mathrm{m}^{3}\right)$ & $53.45 \pm 21.8$ & 2.24 & 37.44 & 45.54 & 68.33 & 188.22 \\
\hline Temperature $\left({ }^{\circ} \mathrm{C}\right)$ & $14.8 \pm 9.8$ & -15.1 & 6.7 & 15 & 23.9 & 33 \\
\hline Humidity (\%) & $44.9 \pm 21.1$ & 12 & 26 & 42 & 61 & 99 \\
\hline \multicolumn{7}{|c|}{ Respiratory admissions per day } \\
\hline All & $7.1 \pm 5.5$ & 0 & 3 & 6 & 10 & 38 \\
\hline Male & $4.1 \pm 3.4$ & 0 & 1 & 3 & 6 & 23 \\
\hline Female & $3.01 \pm 2.7$ & 0 & 1 & 2 & 5 & 22 \\
\hline 0-18 year-olds & $3.7 \pm 3.9$ & 0 & 0 & 3 & 6 & 21 \\
\hline $19-60$ year-olds & $1.2 \pm 1.4$ & 0 & 0 & 1 & 2 & 18 \\
\hline $60+$ year-olds & $2.2 \pm 1.8$ & 0 & 1 & 2 & 3 & 13 \\
\hline
\end{tabular}

$0.02)$, and $\mathrm{PM}_{2.5}$ at lag 7 day $(p=0.03)$ in females. Whereas males had a higher risk of respiratory admissions than females with an increase in $\mathrm{PM}_{10}$ at lag 0 day $(p=0.003), \mathrm{CO}$ at lag 0 day $(p=0.02), \mathrm{SO}_{2}$ at lag 0 day $(p=0.042), \mathrm{NO}_{2}$ at lag $0(p=0.01)$ and at lag 6 days $(p=$ $0.046)$, and $\mathrm{O}_{3}$ at lag 1 day $(P=0.001)$, in regard to respiratory hospital admissions.

Table 4 and Fig. 4 show the exposure-response relationship between air pollutants and respiratory hospital admissions for different lags, in single-pollutant models among different age groups. Positive and statistically significant associations were observed with $\mathrm{NO}_{2}(P=$ $0.005), \mathrm{PM}_{10}(p=0.009), \mathrm{PM}_{2.5}(P=0.03)$ and $\mathrm{CO}(p=$ $0.002)$ at lag 0 day, the corresponding RRs and $(95 \% \mathrm{CI})$ were 1.06(1.02-1.10), 1.015(1.005-1.024), 1.03(1.005-1.06) and $1.15(1.06-1.024)$ per $10 \mu \mathrm{g} / \mathrm{m}^{3}$ increase in the concentrations of pollutants or $1 \mathrm{mg} / \mathrm{m}^{3}$ increase in $\mathrm{CO}$ in elderly (aged $>60$ ) group. Negative significant associations were observed with $\mathrm{O}_{3}$ and respiratory hospital admissions $(P=0.001)$ in the elderly (aged $>60)$ group. The effect of $\mathrm{CO}, \mathrm{NO}_{2}$ and $\mathrm{PM}_{2.5}$, was strongest in the elderly (aged $>60)$ group. $\mathrm{CO}(P=0.044) \mathrm{O}_{3}(P=0.004)$ and $\mathrm{PM}_{10}(P=0.025)$ also showed positive significant association in aged $<60$ years, group.

Fig. 5 shows the exposure-response relationship between air pollutants and respiratory hospital admissions for every $10 \mu \mathrm{g} / \mathrm{m}^{3}$ increase in pollutant's concentrations and every $1 \mathrm{mg} / \mathrm{m}^{3}$ increase in $\mathrm{CO}$ concentrations; in two-pollutant models. Almost all exposure-response relationship between air pollutants and respiratory hospital admissions were relatively constant after adjusting for other air pollutants. The association between respiratory hospital admissions and $\mathrm{SO}_{2}$ tended to be significant after adjustment for $\mathrm{PM}_{2.5}(\mathrm{RR}=1.009,95 \% \mathrm{Cl}$ : 0.999 1.019), but not other pollutants. As for $\mathrm{NO}_{2}$, when adjusted for $\mathrm{CO}$ and $\mathrm{O}_{3}$, the estimated effect decreased to $(\mathrm{RR}=1.023,95 \% \mathrm{Cl}: 0.993-1.053)$ and $(\mathrm{RR}=1.028,95 \% \mathrm{Cl}$ : $0.999-1.06)$, respectively and were insignificant.

\section{Discussion}

The present study showed that, $\mathrm{CO}, \mathrm{PM}_{10}$ and $\mathrm{NO}_{2}$ had a positive association with respiratory hospital admissions. However, the association between other pollutants including $\mathrm{SO}_{2}$ and $\mathrm{PM}_{2.5}$ and daily hospital respiratory admissions was only significant in males and the elderly at lag 0 day, respectively. Unexpectedly, $\mathrm{O}_{3}$ had a negative association with respiratory hospital admissions.

$\mathrm{PM}_{10}$ has been shown to produce oxidative stress and increase inflammatory markers in exposed subjects [41]. In this study, the effect estimate was 1\% (95\%CI: 1.0041.017), increase in respiratory hospital admissions per $10 \mu \mathrm{g} / \mathrm{m}^{3}$ increase in $\mathrm{PM}_{10}$. Several studies have suggested that ambient $\mathrm{PM}_{10}$ is effective on respiratory hospital admissions. For example, a study by Atkinson et al in 2001 investigated the adjusted short-term health effects of ambient particles, in eight European cities, and found a $0.9 \%$ increased risk of total respiratory disease hospitalizations for each $10 \mu \mathrm{g} / \mathrm{m}^{3}$ increase in $\mathrm{PM}_{10}$ [42]. Ma et al in 2016 in Lanzhou, China estimated the risk of emergency room visits for respiratory diseases associated with exposure to ambient air pollution in the spring dust storm season and concluded that ER visits for respiratory diseases increased by $1.14 \%$ for each $10 \mu \mathrm{g} / \mathrm{m}^{3}$ increase in $\mathrm{PM}_{10}$ at lag-3 day [21]. Other studies [43] from Korea, [13] the US, [9] Italy and [20] China, reported that a $10 \mu \mathrm{g} / \mathrm{m}^{3}$ increase in $\mathrm{PM}_{10}$ was associated with a 


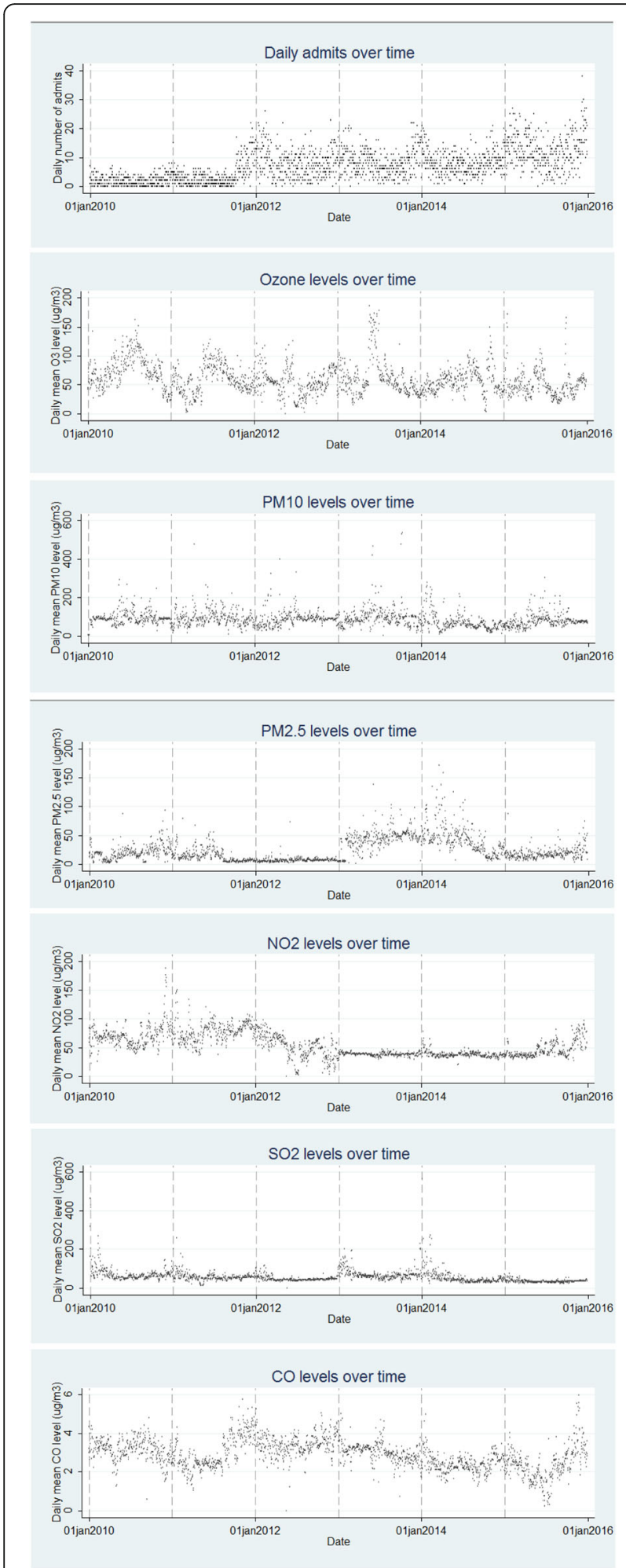

Fig. 1 Daily total respiratory hospital admissions and air pollutants during the study period
$0.77 \%, 3.2 \%, 0.77 \%$ and $0.2 \%$ increase in hospitalization for respiratory diseases. In contrast to this study, Shahi et al in 2014 reported no increase in respiratory diseases hospitalizations related to $\mathrm{PM}_{10}$ in Tehran, Iran. However, this insignificancy can be due to the short study period (2012-2013) and use of limited data. In Shahi's study data from only one hospital was used. Meanwhile, the mean annual concentration of $\mathrm{PM}_{10}$ was $64.72 \mu \mathrm{g} /$ $\mathrm{m}^{3}$ [23] which was less than the current study and Tao [20] $\left(196.63 \mu \mathrm{g} / \mathrm{m}^{3}\right)$ and $\mathrm{Ma}$ [21] $\left(159.2 \mu \mathrm{g} / \mathrm{m}^{3}\right)$ studies.

In this study, no positive association was found between $\mathrm{O}_{3}$ or $\mathrm{PM}_{2.5}$ levels and respiratory hospitalizations on the same day (at lag 0 day). Also, we observed a protective effect of $\mathrm{O}_{3}$ on respiratory hospitalizations after adjustment for other air pollutants. But the lag model showed that ozone had a significant adverse effect on respiratory admissions at lag 1 day. A study done by Wilson et al from Europe in 2005 reported that increases in $\mathrm{O}_{3}$, was not positively associated with the number of all respiratory hospitalizations in Portland and Manchester, UK [19]. Another study done by Phung et al investigated air pollution and the risk of respiratory and cardiovascular hospitalizations in Vietnam and did not show any significant association between $\mathrm{O}_{3}$ and respiratory hospitalizations [36]. Also Fung et al study in 2005 in Ontario, Canada did not find any association between $\mathrm{O}_{3}$ and respiratory diseases admissions either [6]. On the other hand, some studies have suggested a negative effect of ozone on respiratory hospital admissions $[13,44,45]$. Altogether, the inconsistency in effects of ozone on respiratory diseases admissions may be dependent on its concentration or patient's characteristics (such as age, sex, occupation or poverty) [46]. For example, despite the higher ozone concentration during summer, more people use air conditioners that may reduce the effect of ozone on health. However, more studies are needed to clarify these contradictory results.

The results of this study were also comparable to Slaughter et al study from the US, that did not find a significant associated between $\mathrm{PM}_{2.5}$ levels and respiratory hospital admissions in Spokane, Washington [47] either. However, $\mathrm{PM}_{2.5}$ has shown significant associations with respiratory hospital admissions in some other studies. $\mathrm{Xu}$ et al explored the association between fine particulate air pollution $\left(\mathrm{PM}_{2.5}\right)$ and respiratory hospital emergency room visits in Beijing, China and found a positive association between them at different lags [48]. Zanobetti et al in 26 US communities explored the association between fine particulate air pollution $\left(\mathrm{PM}_{2.5}\right)$ and cause-specific respiratory emergency admissions and found a $10 \mu \mathrm{g} / \mathrm{m}^{3}$ increase in 2-day averaged $\mathrm{PM}_{2.5^{-}}$ concentration was associated with a $2.07 \%$ increase in respiratory admissions [17]. Also Dominic et al's study in 204 US counties showed a significant positive 
Table 2 RRs (95\% Cls) of respiratory admissions with an increase of $10 \mu \mathrm{g} / \mathrm{m}^{3}$ in air pollutants (and $1 \mathrm{mg} / \mathrm{m}^{3}$ in CO) according to single lag, adjusted unconstrained and constrained DLM models for each air pollutant

\begin{tabular}{|c|c|c|c|c|c|c|c|}
\hline $\begin{array}{l}\text { Pollutant } \\
\text { All: }\end{array}$ & Lag & $\begin{array}{l}\text { Lag terms model one at } \\
\text { a time RR }(95 \% \mathrm{Cl})\end{array}$ & $p$-value & $\begin{array}{l}\text { Adjusted unconstrained DLM } \\
\text { RR }(95 \% \mathrm{Cl})\end{array}$ & $p$-value & $\begin{array}{l}\text { Adjusted constrained DLM } \\
\text { RR }(95 \% \mathrm{Cl})\end{array}$ & $p$-value \\
\hline \multirow[t]{8}{*}{$\mathrm{SO}_{2}$} & $\operatorname{Lag} 0$ & $1.01(0.999-1.02)$ & 0.06 & $1.01(0.998-1.02)$ & 0.11 & $1.01(0.998-1.02)$ & 0.12 \\
\hline & Lag 1 & $1.001(0.99-1.01)$ & 0.74 & $0.999(0.989-1.01)$ & 0.81 & $1.001(0.995-1.007)$ & 0.87 \\
\hline & $\operatorname{Lag} 2$ & $1.003(0.994-1.012)$ & 0.48 & $0.999(0.989-1.01)$ & 0.73 & $1.001(0.995-1.007)$ & 0.87 \\
\hline & Lag 3 & $1.01(0.997-1.014)$ & 0.22 & $1.006(0.996-1.017)$ & 0.29 & $1.0005(0.997-1.004)$ & 0.71 \\
\hline & $\operatorname{Lag} 4$ & $1.003(0.995-1.01)$ & 0.46 & $1.002(0.99-1.013)$ & 0.40 & $1.0005(0.997-1.004)$ & 0.71 \\
\hline & $\operatorname{Lag} 5$ & $0.99(0.986-1.004)$ & 0.26 & $0.993(0.98-1.004)$ & 0.27 & $1.0005(0.997-1.004)$ & 0.71 \\
\hline & $\operatorname{Lag} 6$ & $0.996(0.99-1.005)$ & 0.42 & 0.999(0.987-1.01) & 0.94 & $1.0005(0.997-1.004)$ & 0.71 \\
\hline & $\operatorname{Lag} 7$ & $1.0002(0.99-1.01)$ & 0.96 & $1.005(0.994-1.017)$ & 0.71 & $1.0005(0.997-1.004)$ & 0.71 \\
\hline \multirow[t]{8}{*}{$\mathrm{CO}$} & $\operatorname{Lag} 0$ & $1.07(1.02-1.12)$ & 0.002 & $1.09(1.04-1.14)$ & 0.005 & $1.09(1.04-1.14)$ & 0.006 \\
\hline & Lag 1 & $0.997(0.955-1.04)$ & 0.89 & $0.99(0.94-1.04)$ & 0.39 & $0.975(0.95-1.005)$ & 0.12 \\
\hline & $\operatorname{Lag} 2$ & $0.96(0.92-0.999)$ & 0.05 & $0.97(0.92-1.02)$ & 0.43 & $0.975(0.95-1.005)$ & 0.12 \\
\hline & Lag 3 & $0.95(0.90-0.997)$ & 0.035 & $0.98(0.93-1.03)$ & 0.55 & $0.984(0.97-0.998)$ & 0.04 \\
\hline & $\operatorname{Lag} 4$ & $0.95(0.91-0.995)$ & 0.03 & $0.95(0.90-1.004)$ & 0.07 & $0.984(0.97-0.998)$ & 0.04 \\
\hline & $\operatorname{Lag} 5$ & $0.99(0.94-1.04)$ & 0.71 & $1.04(0.98-1.10)$ & 0.19 & $0.984(0.97-0.998)$ & 0.04 \\
\hline & $\operatorname{Lag} 6$ & $0.95(0.91-0.994)$ & 0.025 & $0.954(0.90-1.01)$ & 0.08 & $0.984(0.97-0.998)$ & 0.04 \\
\hline & $\operatorname{Lag} 7$ & $0.98(0.94-1.02)$ & 0.28 & $1.004(0.95-1.06)$ & 0.97 & $0.984(0.97-0.998)$ & 0.04 \\
\hline \multirow[t]{8}{*}{$\mathrm{NO}_{2}$} & $\operatorname{Lag} 0$ & $1.01(0.99-1.03)$ & 0.29 & $1.032(1.003-1.06)$ & 0.038 & $1.04(1.01-1.07)$ & 0.011 \\
\hline & Lag 1 & $0.99(0.97-1.01)$ & 0.45 & $0.99(0.96-1.02)$ & 0.63 & $0.976(0.96-0.99)$ & 0.01 \\
\hline & $\operatorname{Lag} 2$ & $0.975(0.95-0.996)$ & 0.02 & $0.975(0.94-1.01)$ & 0.14 & $0.976(0.96-0.99)$ & 0.01 \\
\hline & Lag 3 & $0.98(0.96-0.999)$ & 0.04 & $0.98(0.95-1.01)$ & 0.51 & $1.003(0.996-1.01)$ & 0.40 \\
\hline & $\operatorname{Lag} 4$ & $0.995(0.97-1.02)$ & 0.63 & $0.99(0.96-1.02)$ & 0.74 & $1.003(0.996-1.01)$ & 0.40 \\
\hline & $\operatorname{Lag} 5$ & $1.015(0.99-1.04)$ & 0.16 & $1.027(0.993-1.06)$ & 0.21 & $1.003(0.996-1.01)$ & 0.40 \\
\hline & $\operatorname{Lag} 6$ & $1.01(0.99-1.03)$ & 0.30 & $1.025(0.99-1.06)$ & 0.29 & $1.003(0.996-1.01)$ & 0.40 \\
\hline & $\operatorname{Lag} 7$ & $0.995(0.974-1.016)$ & 0.66 & $0.98(0.95-1.01)$ & 0.27 & $1.003(0.996-1.01)$ & 0.40 \\
\hline \multirow[t]{8}{*}{$\mathrm{O}_{3}$} & $\operatorname{Lag} 0$ & $0.995(0.98-1.01)$ & 0.33 & $0.975(0.96-0.99)$ & 0.002 & 0.98(0.97-0.99) & 0.008 \\
\hline & Lag 1 & $1.01(1.002-1.02)$ & 0.02 & $1.03(1.01-1.05)$ & 0.001 & $1.012(1.004-1.02)$ & 0.01 \\
\hline & $\operatorname{Lag} 2$ & $1.007(0.996-1.017)$ & 0.20 & $0.99(0.97-1.01)$ & 0.31 & $1.012(1.004-1.02)$ & 0.01 \\
\hline & Lag 3 & $1.01(1.0002-1.02)$ & 0.046 & $1.005(0.99-1.02)$ & 0.52 & $1.001(0.997-1.004)$ & 0.33 \\
\hline & $\operatorname{Lag} 4$ & $1.01(0.998-1.02)$ & 0.10 & $1.001(0.98-1.02)$ & 0.60 & $1.001(0.997-1.004)$ & 0.33 \\
\hline & $\operatorname{Lag} 5$ & $1.009(0.998-1.02)$ & 0.09 & $1.01(0.99-1.03)$ & 0.23 & $1.001(0.997-1.004)$ & 0.33 \\
\hline & $\operatorname{Lag} 6$ & $1.005(0.99-1.02)$ & 0.39 & $0.999(0.98-1.016)$ & 0.99 & $1.001(0.997-1.004)$ & 0.33 \\
\hline & $\operatorname{Lag} 7$ & $1.0004(0.99-1.01)$ & 0.94 & $0.993(0.978-1.008)$ & 0.29 & $1.001(0.997-1.004)$ & 0.33 \\
\hline \multirow[t]{8}{*}{$\mathrm{PM}_{2.5}$} & $\operatorname{Lag} 0$ & $1.007(0.99-1.02)$ & 0.46 & $1.01(0.99-1.03)$ & 0.47 & $1.01(0.99-1.03)$ & 0.37 \\
\hline & Lag 1 & $0.998(0.98-1.02)$ & 0.89 & $0.998(0.98-1.02)$ & 0.91 & $0.99(0.98-1.002)$ & 0.11 \\
\hline & $\operatorname{Lag} 2$ & $0.99(0.97-1.01)$ & 0.18 & $0.99(0.97-1.01)$ & 0.16 & $0.99(0.98-1.002)$ & 0.11 \\
\hline & $\operatorname{Lag} 3$ & $1.001(0.98-1.02)$ & 0.92 & $1.01(0.99-1.03)$ & 0.85 & $1.006(1.001-1.01)$ & 0.03 \\
\hline & $\operatorname{Lag} 4$ & $1.003(0.99-1.02)$ & 0.71 & $1.002(0.98-1.022)$ & 0.64 & $1.006(1.001-1.01)$ & 0.03 \\
\hline & $\operatorname{Lag} 5$ & $1.007(0.99-1.02)$ & 0.39 & $1.004(0.984-1.025)$ & 0.28 & $1.006(1.001-1.01)$ & 0.03 \\
\hline & $\operatorname{Lag} 6$ & $1.01(0.99-1.03)$ & 0.29 & $0.998(0.98-1.02)$ & 0.48 & $1.006(1.001-1.01)$ & 0.03 \\
\hline & $\operatorname{Lag} 7$ & $1.02(1.007-1.04)$ & 0.005 & $1.024(1.005-1.043)$ & 0.03 & $1.006(1.001-1.01)$ & 0.03 \\
\hline
\end{tabular}


Table 2 RRs ( $95 \%$ Cls) of respiratory admissions with an increase of $10 \mu \mathrm{g} / \mathrm{m}^{3}$ in air pollutants (and $1 \mathrm{mg} / \mathrm{m}^{3}$ in CO) according to single lag, adjusted unconstrained and constrained DLM models for each air pollutant (Continued)

\begin{tabular}{llllllll}
\hline $\mathrm{PM}_{10}$ & Lag 0 & $1.004(0.999-1.01)$ & 0.12 & $1.01(1.004-1.017)$ & 0.002 & $1.01(1.002-1.02)$ & 0.002 \\
& Lag 1 & $0.995(0.99-1.001)$ & 0.09 & $0.99(0.983-0.998)$ & 0.02 & $0.995(0.99-0.998)$ & 0.01 \\
$\operatorname{Lag} 2$ & $0.995(0.99-1.001)$ & 0.13 & $0.999(0.99-1.007)$ & 0.95 & $0.995(0.99-0.998)$ & 0.01 \\
$\operatorname{Lag} 3$ & $0.997(0.99-1.003)$ & 0.36 & $0.999(0.99-1.007)$ & 0.85 & $1.001(0.998-1.002)$ & 0.57 \\
$\operatorname{Lag} 4$ & $0.996(0.99-1.002)$ & 0.25 & $0.996(0.988-1.004)$ & 0.33 & $1.001(0.998-1.002)$ & 0.57 \\
$\operatorname{Lag} 5$ & $0.999(0.99-1.005)$ & 0.83 & $0.999(0.99-1.007)$ & 0.61 & $1.001(0.998-1.002)$ & 0.57 \\
$\operatorname{Lag} 6$ & $1.003(0.997-1.009)$ & 0.31 & $1.003(0.995-1.01)$ & 0.46 & $1.001(0.998-1.002)$ & 0.57 \\
$\operatorname{Lag} 7$ & $1.004(0.998-1.01)$ & 0.22 & $1.001(0.994-1.008)$ & 0.70 & $1.001(0.998-1.002)$ & 0.57 \\
\hline
\end{tabular}

association between $\mathrm{PM}_{2.5}$ and respiratory hospital admissions [8]. The association between $\mathrm{PM}_{2.5}$ and daily hospital respiratory admissions in older adults $(60+$ year-olds) was significant at lag-0 in the present study where the mean annual concentration of $\mathrm{PM}_{2.5}$ was equal to $24.3 \mu \mathrm{g} / \mathrm{m}^{3}$ and is consistent with $\mathrm{Xu}$ et al's study with mean annual concentration of $\mathrm{PM}_{2.5}$ equal $102.1 \mu \mathrm{g} / \mathrm{m}^{3}$ [48] from China.

In the current study, $\mathrm{CO}$ showed a significant association with respiratory hospital admissions. Several other world studies are in line with these results [15, 23, 49]. Samoli et al's study in London, UK reported evidence for a consistent adverse effects of short-term $\mathrm{CO}$ exposures on adult respiratory hospital admissions [49]. In another study done by Shahi et al in 2014, in Tehran, Iran; total respiratory diseases hospitalizations increased by $4 \%$ for each $10 \mu \mathrm{g} / \mathrm{m}^{3}$ increase in CO levels in urban areas [23]. A quantitative systematic review including 134 papers and estimates from 173 cities also resulted in a significant association between the $\mathrm{CO}$ levels and respiratory hospital admissions [50]. However, a study by Slaughter et al from the US, found no significant association between $\mathrm{CO}$ levels and respiratory hospital admissions in Spokane, Washington [47].

The results of this study were comparable to Chen et al study, in 2010 from China, that did not find a significant association between $\mathrm{SO}_{2}$ levels and respiratory hospital admissions in Shanghai [51]. Also another study done by Shahi et al in Tehran, Iran did not show an increase in respiratory diseases hospitalizations with increases in $\mathrm{SO}_{2}$ concentrations [23] either. In these two mention studies, the mean annual concentration of $\mathrm{SO}_{2}$ were $56 \mu \mathrm{g} / \mathrm{m}^{3}$ [51] and $32.22 \mu \mathrm{g} / \mathrm{m}^{3}$ [23], respectively; which are equal or lower than the $\mathrm{SO}_{2}$ concentrations in our study $\left(54.83 \mu \mathrm{g} / \mathrm{m}^{3}\right)$. However, these insignificant results can be due to the short study durations (2005-

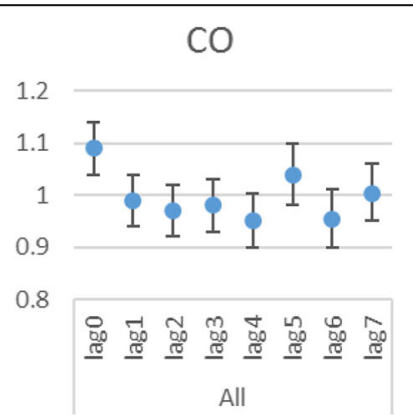

PM10

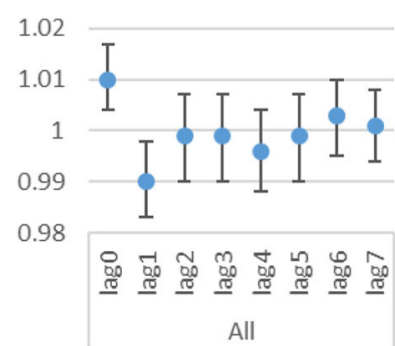

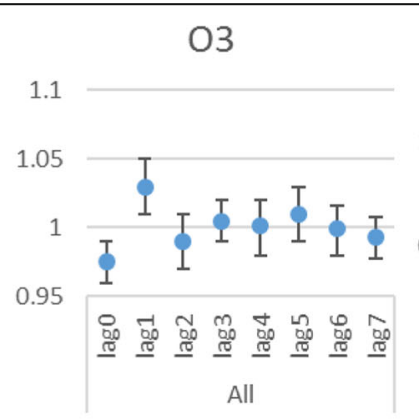

$\mathrm{SO} 2$

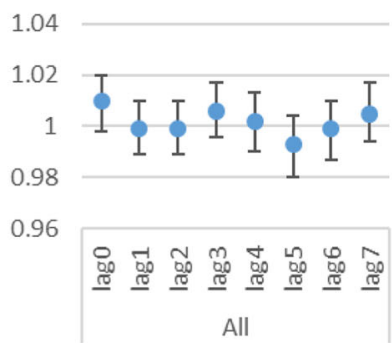

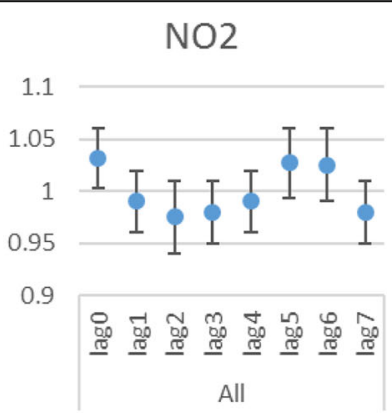

PM2.5

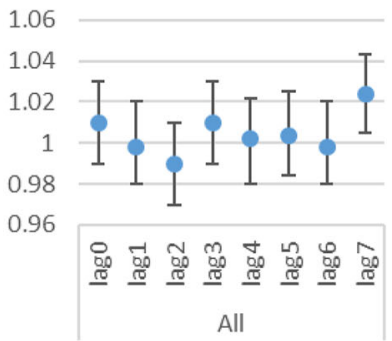

Fig. 2 RRs ( $95 \% \mathrm{Cls}$ ) of respiratory admissions with an increase of $10 \mu \mathrm{g} / \mathrm{m}^{3}$ in air pollutants (and $1 \mathrm{mg} / \mathrm{m}^{3} \mathrm{in} \mathrm{CO}$ ) according to adjusted unconstrained models 
Table 3 RRs (95\% Cls) of respiratory admissions with an increase of $10 \mu \mathrm{g} / \mathrm{m}^{3}$ in air pollutants (and $1 \mathrm{mg} / \mathrm{m}^{3}$ in CO) according to single lag, adjusted unconstrained and constrained DLM models for each air pollutant in both genders

\begin{tabular}{|c|c|c|c|c|c|c|c|c|}
\hline Pollutants & & Lag & $\begin{array}{l}\text { Lag terms model one at } \\
\text { a time RR }(95 \% \mathrm{Cl})\end{array}$ & $p$-value & $\begin{array}{l}\text { Adjusted unconstrained } \\
\text { DLM RR }(95 \% \mathrm{Cl})\end{array}$ & $p$-value & $\begin{array}{l}\text { Adjusted constrained } \\
\text { DLM RR }(95 \% \mathrm{CI})\end{array}$ & $p$-value \\
\hline \multirow[t]{44}{*}{ Male } & \multirow[t]{8}{*}{$\mathrm{SO}_{2}$} & $\operatorname{Lag} 0$ & $1.01(1.001-1.02)$ & 0.04 & $1.012(1.0001-1.024)$ & 0.042 & $1.01(1.001-1.02)$ & 0.04 \\
\hline & & Lag 1 & $1.003(0.992-1.013)$ & 0.57 & $1.001(0.988-1.014)$ & 0.77 & $1.0001(0.992-1.01)$ & 0.91 \\
\hline & & $\operatorname{Lag} 2$ & $1.002(0.99-1.013)$ & 0.65 & 0.998(0.985-1.012) & 0.70 & $1.0001(0.992-1.01)$ & 0.91 \\
\hline & & $\operatorname{Lag} 3$ & $1.002(0.991-1.012)$ & 0.72 & $1.003(0.99-1.016)$ & 0.74 & $1.001(0.997-1.005)$ & 0.44 \\
\hline & & Lag 4 & $1.005(0.99-1.015)$ & 0.31 & $1.005(0.99-1.02)$ & 0.21 & $1.001(0.997-1.005)$ & 0.44 \\
\hline & & $\operatorname{Lag} 5$ & 0.998(0.987-1.009) & 0.75 & $0.994(0.98-1.01)$ & 0.47 & $1.001(0.997-1.005)$ & 0.44 \\
\hline & & $\operatorname{Lag} 6$ & $1.0003(0.99-1.01)$ & 0.96 & $1.005(0.99-1.02)$ & 0.56 & $1.001(0.997-1.005)$ & 0.44 \\
\hline & & $\operatorname{Lag} 7$ & 0.998(0.987-1.009) & 0.73 & 0.999(0.985-1.013) & 0.87 & $1.001(0.997-1.005)$ & 0.44 \\
\hline & \multirow[t]{8}{*}{$\mathrm{CO}$} & $\operatorname{Lag} 0$ & $1.07(1.02-1.13)$ & 0.007 & $1.09(1.03-1.15)$ & 0.02 & $1.085(1.02-1.15)$ & 0.03 \\
\hline & & Lag 1 & $1.004(0.95-1.06)$ & 0.88 & $0.98(0.92-1.04)$ & 0.32 & $0.98(0.94-1.02)$ & 0.40 \\
\hline & & $\operatorname{Lag} 2$ & $0.98(0.93-1.03)$ & 0.48 & $0.985(0.92-1.05)$ & 0.98 & $0.98(0.94-1.02)$ & 0.40 \\
\hline & & Lag 3 & $0.95(0.90-1.004)$ & 0.07 & $0.965(0.90-1.03)$ & 0.33 & $0.985(0.97-1.002)$ & 0.053 \\
\hline & & $\operatorname{Lag} 4$ & $0.97(0.92-1.02)$ & 0.25 & $0.964(0.90-1.03)$ & 0.25 & $0.985(0.97-1.002)$ & 0.053 \\
\hline & & Lag 5 & $1.01(0.96-1.06)$ & 0.63 & $1.06(0.995-1.13)$ & 0.12 & $0.985(0.97-1.002)$ & 0.053 \\
\hline & & $\operatorname{Lag} 6$ & $0.97(0.92-1.02)$ & 0.19 & $0.976(0.91-1.04)$ & 0.51 & $0.985(0.97-1.002)$ & 0.053 \\
\hline & & $\operatorname{Lag} 7$ & $0.945(0.898-0.994)$ & 0.03 & $0.955(0.90-1.01)$ & 0.06 & $0.985(0.97-1.002)$ & 0.053 \\
\hline & \multirow[t]{8}{*}{$\mathrm{NO}_{2}$} & $\operatorname{Lag} 0$ & $1.018(0.993-1.044)$ & 0.16 & $1.05(1.01-1.08)$ & 0.01 & $1.048(1.014-1.08)$ & 0.01 \\
\hline & & Lag 1 & $0.99(0.965-1.017)$ & 0.51 & $0.97(0.93-1.01)$ & 0.21 & $0.97(0.95-0.99)$ & 0.02 \\
\hline & & $\operatorname{Lag} 2$ & 0.98(0.955-1.006) & 0.14 & $0.98(0.94-1.02)$ & 0.50 & $0.97(0.95-0.99)$ & 0.02 \\
\hline & & Lag 3 & $0.98(0.956-1.006)$ & 0.14 & $0.98(0.94-1.02)$ & 0.58 & $1.004(0.996-1.012)$ & 0.32 \\
\hline & & $\operatorname{Lag} 4$ & $0.995(0.97-1.02)$ & 0.72 & $0.99(0.95-1.03)$ & 0.69 & $1.004(0.996-1.012)$ & 0.32 \\
\hline & & Lag 5 & $1.017(0.99-1.044)$ & 0.20 & $1.02(0.98-1.06)$ & 0.39 & $1.004(0.996-1.012)$ & 0.32 \\
\hline & & $\operatorname{Lag} 6$ & $1.018(0.993-1.045)$ & 0.17 & $1.04(1.001-1.08)$ & 0.046 & $1.004(0.996-1.012)$ & 0.32 \\
\hline & & $\operatorname{Lag} 7$ & 0.995 (0.97-1.02) & 0.70 & $0.972(0.94-1.006)$ & 0.13 & $1.004(0.996-1.012)$ & 0.32 \\
\hline & \multirow[t]{8}{*}{$\mathrm{O}_{3}$} & $\operatorname{Lag} 0$ & $0.994(0.98-1.007)$ & 0.35 & $0.972(0.956-0.988)$ & 0.001 & $0.976(0.96-0.99)$ & 0.008 \\
\hline & & Lag 1 & $1.013(1.001-1.026)$ & 0.035 & $1.04(1.02-1.06)$ & 0.000 & $1.02(1.01-1.03)$ & 0.008 \\
\hline & & $\operatorname{Lag} 2$ & $1.007(0.994-1.02)$ & 0.30 & $0.99(0.97-1.01)$ & 0.19 & $1.02(1.01-1.03)$ & 0.008 \\
\hline & & $\operatorname{Lag} 3$ & $1.013(1.001-1.026)$ & 0.034 & $1.01(0.99-1.03)$ & 0.24 & $0.999(0.996-1.004)$ & 0.71 \\
\hline & & $\operatorname{Lag} 4$ & $1.007(0.995-1.02)$ & 0.28 & $0.999(0.98-1.02)$ & 0.73 & 0.999(0.996-1.004) & 0.71 \\
\hline & & $\operatorname{Lag} 5$ & $1.005(0.99-1.02)$ & 0.40 & $1.003(0.983-1.023)$ & 0.77 & $0.999(0.996-1.004)$ & 0.71 \\
\hline & & $\operatorname{Lag} 6$ & $1.002(0.99-1.01)$ & 0.79 & $0.998(0.978-1.018)$ & 0.74 & $0.999(0.996-1.004)$ & 0.71 \\
\hline & & $\operatorname{Lag} 7$ & $1.0001(0.99-1.01)$ & 0.99 & $0.997(0.98-1.014)$ & 0.80 & $0.999(0.996-1.004)$ & 0.71 \\
\hline & \multirow[t]{8}{*}{$\mathrm{PM}_{2.5}$} & Lag 0 & $1.007(0.99-1.03)$ & 0.50 & $1.013(0.99-1.036)$ & 0.30 & $1.02(0.99-1.04)$ & 0.2 \\
\hline & & Lag 1 & $0.99(0.97-1.01)$ & 0.42 & $0.998(0.97-1.02)$ & 0.91 & $0.98(0.97-0.99)$ & 0.005 \\
\hline & & $\operatorname{Lag} 2$ & $0.97(0.95-0.99)$ & 0.005 & $0.97(0.95-0.99)$ & 0.014 & $0.98(0.97-0.99)$ & 0.005 \\
\hline & & $\operatorname{Lag} 3$ & $0.98(0.96-1.004)$ & 0.10 & $0.99(0.97-1.01)$ & 0.23 & $1.005(0.998-1.01)$ & 0.21 \\
\hline & & $\operatorname{Lag} 4$ & $1.003(0.98-1.02)$ & 0.70 & $1.016(0.992-1.04)$ & 0.15 & $1.005(0.998-1.01)$ & 0.21 \\
\hline & & Lag 5 & $0.997(0.98-1.01)$ & 0.80 & $0.995(0.97-1.02)$ & 0.92 & $1.005(0.998-1.01)$ & 0.21 \\
\hline & & $\operatorname{Lag} 6$ & 1.01 (0.99-1.03) & 0.35 & $1.01(0.99-1.03)$ & 0.82 & $1.005(0.998-1.01)$ & 0.21 \\
\hline & & $\operatorname{Lag} 7$ & $1.02(1.002-1.04)$ & 0.03 & $1.02(0.997-1.04)$ & 0.15 & $1.005(0.998-1.01)$ & 0.21 \\
\hline & \multirow[t]{4}{*}{$\mathrm{PM}_{10}$} & $\operatorname{Lag} 0$ & $1.005(0.998-1.01)$ & 0.15 & $1.012(1.004-1.02)$ & 0.003 & $1.01(1.003-1.02)$ & 0.003 \\
\hline & & $\operatorname{Lag} 1$ & $0.99(0.98-0.999)$ & 0.03 & $0.99(0.98-0.997)$ & 0.01 & $0.992(0.988-0.997)$ & 0.005 \\
\hline & & $\operatorname{Lag} 2$ & $0.99(0.98-0.999)$ & 0.03 & 0.998(0.989-1.007) & 0.94 & $0.992(0.988-0.997)$ & 0.005 \\
\hline & & $\operatorname{Lag} 3$ & $0.995(0.99-1.002)$ & 0.14 & $0.997(0.988-1.006)$ & 0.31 & $0.999(0.997-1.001)$ & 0.66 \\
\hline
\end{tabular}


Table 3 RRs ( $95 \%$ Cls) of respiratory admissions with an increase of $10 \mu \mathrm{g} / \mathrm{m}^{3}$ in air pollutants (and $1 \mathrm{mg} / \mathrm{m}^{3}$ in CO) according to single lag, adjusted unconstrained and constrained DLM models for each air pollutant in both genders (Continued)

\begin{tabular}{|c|c|c|c|c|c|c|c|c|}
\hline & & $\operatorname{Lag} 4$ & $0.996(0.99-1.003)$ & 0.30 & $1.0002(0.99-1.01)$ & 0.99 & $0.999(0.997-1.001)$ & 0.66 \\
\hline & & Lag 5 & $0.996(0.99-1.003)$ & 0.30 & 0.998(0.99-1.007) & 0.96 & $0.999(0.997-1.001)$ & 0.66 \\
\hline & & $\operatorname{Lag} 6$ & $0.998(0.99-1.005)$ & 0.62 & $0.998(0.989-1.007)$ & 0.91 & $0.999(0.997-1.001)$ & 0.66 \\
\hline & & $\operatorname{Lag} 7$ & $1.001(0.994-1.008)$ & 0.80 & $1.001(0.993-1.009)$ & 0.79 & $0.999(0.997-1.001)$ & 0.66 \\
\hline \multirow[t]{42}{*}{ Female } & \multirow[t]{8}{*}{$\mathrm{SO}_{2}$} & Lag 0 & $1.005(0.994-1.016)$ & 0.40 & $1.003(0.99-1.017)$ & 0.32 & $1.002(0.99-1.01)$ & 0.58 \\
\hline & & Lag 1 & 0.999(0.988-1.01) & 0.92 & $0.997(0.983-1.01)$ & 0.44 & $1.002(0.99-1.01)$ & 0.87 \\
\hline & & $\operatorname{Lag} 2$ & $1.004(0.993-1.015)$ & 0.48 & $1.001(0.987-1.015)$ & 0.89 & $1.002(0.99-1.01)$ & 0.87 \\
\hline & & Lag 3 & $1.01(0.998-1.02)$ & 0.09 & $1.01(0.997-1.024)$ & 0.15 & $0.999(0.995-1.004)$ & 0.80 \\
\hline & & $\operatorname{Lag} 4$ & $1.001(0.99-1.01)$ & 0.91 & $0.997(0.983-1.01)$ & 0.98 & $0.999(0.995-1.004)$ & 0.80 \\
\hline & & $\operatorname{Lag} 5$ & $0.99(0.98-1.002)$ & 0.11 & 0.99(0.975-1.006) & 0.29 & $0.999(0.995-1.004)$ & 0.80 \\
\hline & & $\operatorname{Lag} 6$ & $0.99(0.98-1.003)$ & 0.13 & $0.99(0.975-1.007)$ & 0.43 & $0.999(0.995-1.004)$ & 0.80 \\
\hline & & $\operatorname{Lag} 7$ & $1.003(0.99-1.014)$ & 0.63 & $1.014(0.998-1.03)$ & 0.41 & $0.999(0.995-1.004)$ & 0.80 \\
\hline & \multirow[t]{8}{*}{$\mathrm{CO}$} & $\operatorname{Lag} 0$ & $1.06(1.006-1.12)$ & 0.03 & $1.09(1.02-1.16)$ & 0.026 & $1.10(1.03-1.17)$ & 0.02 \\
\hline & & Lag 1 & $0.99(0.0 .93-1.05)$ & 0.68 & $1.002(0.93-1.08)$ & 0.76 & $0.97(0.93-1.01)$ & 0.08 \\
\hline & & $\operatorname{Lag} 2$ & $0.93(0.88-0.98)$ & 0.009 & $0.94(0.87-1.01)$ & 0.13 & $0.97(0.93-1.01)$ & 0.08 \\
\hline & & $\operatorname{Lag} 3$ & $0.95(0.90-1.01)$ & 0.12 & $0.995(0.92-1.07)$ & 0.90 & $0.98(0.96-1.002)$ & 0.18 \\
\hline & & $\operatorname{Lag} 4$ & $0.93(0.88-0.98)$ & 0.014 & $0.937(0.87-1.005)$ & 0.08 & $0.98(0.96-1.002)$ & 0.18 \\
\hline & & $\operatorname{Lag} 5$ & $0.965(0.91-1.02)$ & 0.21 & $1.004(0.94-1.07)$ & 0.69 & $0.98(0.96-1.002)$ & 0.18 \\
\hline & & $\operatorname{Lag} 6$ & $0.93(0.88-0.99)$ & 0.017 & $0.927(0.86-0.995)$ & 0.02 & $0.98(0.96-1.002)$ & 0.18 \\
\hline & & $\operatorname{Lag} 7$ & $1.02(0.96-1.08)$ & 0.52 & $1.074(1.006-1.15)$ & 0.02 & $0.98(0.96-1.002)$ & 0.18 \\
\hline & \multirow[t]{8}{*}{$\mathrm{NO}_{2}$} & $\operatorname{Lag} 0$ & $1.003(0.975-1.03)$ & 0.83 & $1.013(0.975-1.05)$ & 0.41 & $1.024(0.99-1.06)$ & 0.14 \\
\hline & & Lag 1 & $0.993(0.965-1.02)$ & 0.60 & $1.015(0.97-1.06)$ & 0.57 & 0.98(0.96-1.003) & 0.08 \\
\hline & & $\operatorname{Lag} 2$ & $0.97(0.942-0.996)$ & 0.025 & $0.965(0.92-1.01)$ & 0.08 & $0.98(0.96-1.003)$ & 0.08 \\
\hline & & Lag 3 & $0.975(0.95-1.002)$ & 0.065 & $0.975(0.93-1.02)$ & 0.64 & $1.002(0.99-1.01)$ & 0.71 \\
\hline & & $\operatorname{Lag} 4$ & $0.994(0.97-1.02)$ & 0.66 & 0.998(0.955-1.04) & 0.89 & $1.002(0.99-1.01)$ & 0.71 \\
\hline & & $\operatorname{Lag} 5$ & $1.013(0.986-1.04)$ & 0.35 & $1.035(0.99-1.08)$ & 0.22 & $1.002(0.99-1.01)$ & 0.71 \\
\hline & & $\operatorname{Lag} 6$ & $1.003(0.975-1.03)$ & 0.85 & $1.001(0.95-1.05)$ & 0.84 & $1.002(0.99-1.01)$ & 0.71 \\
\hline & & $\operatorname{Lag} 7$ & $0.996(0.97-1.02)$ & 0.76 & $0.993(0.956-1.03)$ & 0.89 & $1.002(0.99-1.01)$ & 0.71 \\
\hline & \multirow[t]{8}{*}{$\mathrm{O}_{3}$} & $\operatorname{Lag} 0$ & $0.995(0.98-1.01)$ & 0.55 & $0.98(0.96-1.001)$ & 0.09 & $0.985(0.97-1.004)$ & 0.12 \\
\hline & & Lag 1 & $1.011(0.997-1.025)$ & 0.11 & $1.02(0.998-1.04)$ & 0.11 & $1.007(0.996-1.02)$ & 0.16 \\
\hline & & $\operatorname{Lag} 2$ & $1.007(0.993-1.022)$ & 0.30 & $0.996(0.97-1.02)$ & 0.86 & $1.007(0.996-1.02)$ & 0.16 \\
\hline & & Lag 3 & $1.007(0.993-1.021)$ & 0.32 & 0.995(0.97-1.02) & 0.78 & $1.002(0.997-1.006)$ & 0.20 \\
\hline & & $\operatorname{Lag} 4$ & $1.011(0.997-1.025)$ & 0.11 & $1.003(0.98-1.026)$ & 0.59 & $1.002(0.997-1.006)$ & 0.20 \\
\hline & & Lag 5 & $1.015(1.0004-1.03)$ & 0.044 & $1.02(0.996-1.042)$ & 0.08 & $1.002(0.997-1.006)$ & 0.20 \\
\hline & & $\operatorname{Lag} 6$ & $1.008(0.995-1.02)$ & 0.23 & $1.001(0.98-1.02)$ & 0.67 & $1.002(0.997-1.006)$ & 0.20 \\
\hline & & $\operatorname{Lag} 7$ & $1.001(0.987-1.016)$ & 0.88 & $0.99(0.97-1.01)$ & 0.11 & $1.002(0.997-1.006)$ & 0.20 \\
\hline & \multirow[t]{8}{*}{$\mathrm{PM}_{2.5}$} & $\operatorname{Lag} 0$ & $1.006(0.98-1.03)$ & 0.61 & $1.004(0.98-1.03)$ & 0.98 & $1.004(0.98-1.03)$ & 0.99 \\
\hline & & Lag 1 & $1.01(0.99-1.03)$ & 0.45 & $0.998(0.97-1.026)$ & 0.99 & $1.005(0.99-1.02)$ & 0.58 \\
\hline & & $\operatorname{Lag} 2$ & $1.01(0.99-1.03)$ & 0.30 & $1.01(0.98-1.04)$ & 0.61 & $1.005(0.99-1.02)$ & 0.58 \\
\hline & & $\operatorname{Lag} 3$ & $1.02(1.002-1.05)$ & 0.03 & $1.025(0.999-1.05)$ & 0.07 & $1.008(1.0003-1.015)$ & 0.03 \\
\hline & & $\operatorname{Lag} 4$ & $1.003(0.98-1.03)$ & 0.80 & 0.98(0.954-1.009) & 0.31 & $1.008(1.0003-1.015)$ & 0.03 \\
\hline & & $\operatorname{Lag} 5$ & $1.02(0.998-1.04)$ & 0.064 & $1.02(0.99-1.05)$ & 0.08 & $1.008(1.0003-1.015)$ & 0.03 \\
\hline & & $\operatorname{Lag} 6$ & $1.01(0.99-1.03)$ & 0.46 & $0.99(0.96-1.02)$ & 0.13 & $1.008(1.0003-1.015)$ & 0.03 \\
\hline & & $\operatorname{Lag} 7$ & $1.03(1.005-1.05)$ & 0.016 & $1.03(1.005-1.06)$ & 0.03 & $1.008(1.0003-1.015)$ & 0.03 \\
\hline & \multirow[t]{2}{*}{$\mathrm{PM}_{10}$} & $\operatorname{Lag} 0$ & $1.004(0.996-1.01)$ & 0.30 & $1.01(0.999-1.02)$ & 0.32 & $1.006(0.997-1.01)$ & 0.034 \\
\hline & & Lag 1 & 0.998(0.99-1.006) & 0.70 & $0.994(0.984-1.005)$ & 0.24 & $0.997(0.99-1.003)$ & 0.27 \\
\hline
\end{tabular}


Table 3 RRs ( $95 \%$ Cls) of respiratory admissions with an increase of $10 \mu \mathrm{g} / \mathrm{m}^{3}$ in air pollutants (and $1 \mathrm{mg} / \mathrm{m}^{3}$ in CO) according to single lag, adjusted unconstrained and constrained DLM models for each air pollutant in both genders (Continued)

\begin{tabular}{lllllll} 
Lag 2 & $0.999(0.99-1.01)$ & 0.90 & $1.0007(0.99-1.01)$ & 0.98 & $0.997(0.99-1.003)$ & 0.27 \\
Lag 3 & $1.0006(0.99-1.01)$ & 0.86 & $1.003(0.993-1.013)$ & 0.38 & $1.001(0.999-1.004)$ & 0.13 \\
Lag 4 & $0.996(0.99-1.005)$ & 0.44 & $0.99(0.98-1.001)$ & 0.09 & $1.001(0.999-1.004)$ & 0.13 \\
Lag 5 & $1.003(0.995-1.01)$ & 0.39 & $1.002(0.992-1.011)$ & 0.35 & $1.001(0.999-1.004)$ & 0.13 \\
Lag 6 & $1.01(1.002-1.02)$ & 0.015 & $1.008(0.998-1.02)$ & 0.14 & $1.001(0.999-1.004)$ & 0.13 \\
Lag 7 & $1.007(0.999-1.014)$ & 0.06 & $1.002(0.993-1.01)$ & 0.71 & $1.001(0.999-1.004)$ & 0.13 \\
\hline
\end{tabular}

2007) in Chen et al and (2012-2013) in Shahi et al's two studies and also use of a small sample size. On the other hand, $\mathrm{SO}_{2}$ has shown significant associations with respiratory hospital admissions in some other studies. For example research from Lanzhou, China reported that ER visits for respiratory diseases increased by $2.7 \%$ for each $10 \mu \mathrm{g} / \mathrm{m}^{3}$ increase in $\mathrm{SO}_{2}$ on dust days and by $0.6 \%$ for each $10 \mu \mathrm{g} / \mathrm{m}^{3}$ increase in $\mathrm{SO}_{2}$ on non-dust days [21]. Also other studies from China such as, Zhang et al's study from Beijing, [1], Tao et al's study from Lanzhou [20], and Liu et al's study from Jinan [22], reported that an increase of $10 \mu \mathrm{g} / \mathrm{m}^{3}$ of $\mathrm{SO}_{2}$ corresponded to a $35 \%, 0.5 \%$ and $1.2 \%$ increase of respiratory diseases hospitalizations, respectively. Another study done by Phung et al from Vietnam also found that $\mathrm{SO}_{2}$ was positively associated with the number of respiratory hospital admissions [36]. The mean annual concentration of $\mathrm{SO}_{2}$ in most of the mentioned studies that reported significant association between $\mathrm{SO}_{2}$ levels and respiratory hospital admissions, were higher than the present study. This concentration was reported, $79.09 \mu \mathrm{g} /$ $\mathrm{m}^{3}$ in Lanzhou, China [20], $95.4 \mu \mathrm{g} / \mathrm{m}^{3}$ in Jinan, China [22], and $79.1 \mu \mathrm{g} / \mathrm{m}^{3}$ in western China [52], that almost all of them have, 1.5 to 2 times the $\mathrm{SO}_{2}$ concentration of the present study. However, in our study the association between $\mathrm{SO}_{2}$ and daily hospital respiratory admissions in males was significant, despite the relatively low concentration of $\mathrm{SO}_{2}$.

$\mathrm{NO}_{2}$ is a highly reactive oxidant which contributes to increased susceptibility to respiratory infections [53]. The main sources of ambient $\mathrm{NO}_{2}$ are industrial emissions and motor vehicle exhaust in Arak. The association between $\mathrm{NO}_{2}$ and daily hospital respiratory admissions in this study is consistent with previous studies. Liu et al in Jinan, China showed that an increase of $10 \mu \mathrm{g} / \mathrm{m}^{3}$ of $\mathrm{NO}_{2}$ corresponded to a $2.5 \%$ increase of respiratory disease 
Table 4 RRs (95\% Cls) of respiratory admissions with an increase of $10 \mu \mathrm{g} / \mathrm{m}^{3}$ in air pollutants (and $1 \mathrm{mg} / \mathrm{m}^{3}$ in CO) according to single lag, adjusted unconstrained and constrained DLM models for each air pollutant among two age groups

\begin{tabular}{|c|c|c|c|c|c|c|c|c|}
\hline \multicolumn{2}{|l|}{ Pollutants } & \multirow{2}{*}{$\begin{array}{l}\text { Lag } \\
\text { Lag } 0\end{array}$} & \multirow{2}{*}{$\begin{array}{l}\text { Lag terms model one at } \\
\text { a time RR }(95 \% \mathrm{Cl}) \\
1.01(0.998-1.023)\end{array}$} & \multirow{2}{*}{$\begin{array}{c}p \text {-value } \\
0.087\end{array}$} & \multirow{2}{*}{$\begin{array}{l}\text { Adjusted unconstrained } \\
\text { DLM RR (95\% Cl) } \\
1.011(0.996-1.027)\end{array}$} & \multirow{2}{*}{$\frac{p \text {-value }}{0.10}$} & \multirow{2}{*}{$\begin{array}{l}\text { Adjusted constrained } \\
\text { DLM RR }(95 \% \mathrm{Cl}) \\
1.01(0.995-1.023)\end{array}$} & \multirow{2}{*}{$\begin{array}{c}p \text {-value } \\
0.18\end{array}$} \\
\hline Over60 & $\mathrm{SO}_{2}$ & & & & & & & \\
\hline & & Lag 1 & $1.002(0.99-1.015)$ & 0.72 & $0.997(0.98-1.015)$ & 0.83 & $1.001(0.99-1.01)$ & 0.97 \\
\hline & & $\operatorname{Lag} 2$ & $1.005(0.99-1.02)$ & 0.43 & $0.998(0.98-1.015)$ & 0.79 & $1.001(0.99-1.01)$ & 0.97 \\
\hline & & $\operatorname{Lag} 3$ & $1.008(0.996-1.02)$ & 0.20 & $1.01(0.995-1.027)$ & 0.14 & $1.002(0.997-1.007)$ & 0.49 \\
\hline & & $\operatorname{Lag} 4$ & $1.006(0.994-1.019)$ & 0.31 & $0.999(0.98-1.02)$ & 0.63 & $1.002(0.997-1.007)$ & 0.49 \\
\hline & & $\operatorname{Lag} 5$ & $0.999(0.986-1.012)$ & 0.89 & $0.99(0.97-1.01)$ & 0.45 & $1.002(0.997-1.007)$ & 0.49 \\
\hline & & Lag 6 & $1.001(0.99-1.01)$ & 0.86 & $1.01(0.99-1.03)$ & 0.24 & $1.002(0.997-1.007)$ & 0.49 \\
\hline & & $\operatorname{Lag} 7$ & $0.994(0.98-1.008)$ & 0.43 & $0.994(0.98-1.01)$ & 0.22 & $1.002(0.997-1.007)$ & 0.49 \\
\hline & $\mathrm{CO}$ & $\operatorname{Lag} 0$ & $1.13(1.06-1.20)$ & 0.000 & $1.15(1.06-1.24)$ & 0.002 & $1.15(1.07-1.24)$ & 0.001 \\
\hline & & Lag 1 & $1.05(0.98-1.12)$ & 0.12 & $1.03(0.95-1.12)$ & 0.50 & $0.985(0.94-1.03)$ & 0.68 \\
\hline & & $\operatorname{Lag} 2$ & $0.976(0.91-1.04)$ & 0.47 & $0.965(0.88-1.05)$ & 0.56 & $0.985(0.94-1.03)$ & 0.68 \\
\hline & & Lag 3 & $0.95(0.89-1.01)$ & 0.15 & $0.94(0.86-1.02)$ & 0.12 & $0.982(0.96-1.003)$ & 0.10 \\
\hline & & $\operatorname{Lag} 4$ & $0.974(0.91-1.04)$ & 0.44 & $0.98(0.90-1.06)$ & 0.58 & $0.982(0.96-1.003)$ & 0.10 \\
\hline & & $\operatorname{Lag} 5$ & $0.97(0.91-1.03)$ & 0.39 & $0.986(0.90-1.07)$ & 0.69 & $0.982(0.96-1.003)$ & 0.10 \\
\hline & & Lag 6 & $0.956(0.89-1.02)$ & 0.19 & $0.94(0.86-1.02)$ & 0.26 & $0.982(0.96-1.003)$ & 0.10 \\
\hline & & $\operatorname{Lag} 7$ & $1.03(0.96-1.10)$ & 0.39 & $1.07(0.99-1.16)$ & 0.11 & $0.982(0.96-1.003)$ & 0.10 \\
\hline & $\mathrm{NO}_{2}$ & $\operatorname{Lag} 0$ & $1.003(0.975-1.03)$ & 0.81 & $1.06(1.02-1.10)$ & 0.005 & $1.05(1.01-1.09)$ & 0.009 \\
\hline & & $\operatorname{Lag} 1$ & $0.97(0.94-0.998)$ & 0.036 & $0.95(0.90-1.001)$ & 0.05 & $0.963(0.941-0.986)$ & 0.001 \\
\hline & & $\operatorname{Lag} 2$ & $0.97(0.94-0.998)$ & 0.03 & $0.998(0.95-1.05)$ & 0.80 & $0.963(0.941-0.986)$ & 0.001 \\
\hline & & $\operatorname{Lag} 3$ & $0.96(0.93-0.99)$ & 0.009 & $0.96(0.91-1.01)$ & 0.21 & $1.002(0.99-1.01)$ & 0.47 \\
\hline & & $\operatorname{Lag} 4$ & $0.975(0.95-1.003)$ & 0.08 & $0.96(0.91-1.01)$ & 0.27 & $1.002(0.99-1.01)$ & 0.47 \\
\hline & & $\operatorname{Lag} 5$ & $1.005(0.98-1.03)$ & 0.72 & $1.034(0.98-1.09)$ & 0.32 & $1.002(0.99-1.01)$ & 0.47 \\
\hline & & $\operatorname{Lag} 6$ & $1.01(0.98-1.04)$ & 0.41 & $1.01(0.96-1.06)$ & 0.54 & $1.002(0.99-1.01)$ & 0.47 \\
\hline & & $\operatorname{Lag} 7$ & $1.01(0.98-1.04)$ & 0.36 & $1.016(0.975-1.06)$ & 0.54 & $1.002(0.99-1.01)$ & 0.47 \\
\hline & $\mathrm{O}_{3}$ & Lag 0 & $0.98(0.97-0.99)$ & 0.04 & $0.96(0.94-0.98)$ & 0.001 & $0.97(0.95-0.99)$ & 0.002 \\
\hline & & Lag 1 & $1.01(0.99-1.02)$ & 0.38 & $1.035(1.01-1.06)$ & 0.02 & $1.01(0.999-1.02)$ & 0.11 \\
\hline & & $\operatorname{Lag} 2$ & $0.999(0.98-1.02)$ & 0.99 & $0.987(0.96-1.013)$ & 0.38 & $1.01(0.999-1.02)$ & 0.11 \\
\hline & & Lag 3 & $1.01(0.99-1.03)$ & 0.24 & $1.004(0.98-1.03)$ & 0.73 & $1.003(0.998-1.008)$ & 0.25 \\
\hline & & $\operatorname{Lag} 4$ & $1.01(0.997-1.03)$ & 0.10 & $1.004(0.98-1.03)$ & 0.79 & $1.003(0.998-1.008)$ & 0.25 \\
\hline & & $\operatorname{Lag} 5$ & $1.02(1.003-1.04)$ & 0.017 & $1.027(1.001-1.054)$ & 0.02 & $1.003(0.998-1.008)$ & 0.25 \\
\hline & & $\operatorname{Lag} 6$ & $1.006(0.99-1.023)$ & 0.46 & $0.993(0.967-1.02)$ & 0.31 & $1.003(0.998-1.008)$ & 0.25 \\
\hline & & $\operatorname{Lag} 7$ & $0.998(0.98-1.015)$ & 0.83 & $0.99(0.97-1.01)$ & 0.54 & $1.003(0.998-1.008)$ & 0.25 \\
\hline & $\mathrm{PM}_{2.5}$ & Lag 0 & $1.03(1.002-1.05)$ & 0.03 & $1.03(1.005-1.06)$ & 0.03 & $1.04(1.007-1.06)$ & 0.04 \\
\hline & & Lag 1 & $1.004(0.98-1.03)$ & 0.76 & $0.994(0.96-1.03)$ & 0.81 & $0.98(0.97-1.001)$ & 0.08 \\
\hline & & $\operatorname{Lag} 2$ & $0.99(0.97-1.02)$ & 0.52 & $0.98(0.95-1.01)$ & 0.18 & $0.98(0.97-1.001)$ & 0.08 \\
\hline & & Lag 3 & $1.01(0.99-1.04)$ & 0.30 & $1.01(0.98-1.04)$ & 0.86 & $1.01(1.005-1.02)$ & 0.005 \\
\hline & & $\operatorname{Lag} 4$ & $1.02(0.99-1.04)$ & 0.20 & $1.01(0.98-1.04)$ & 0.58 & $1.01(1.005-1.02)$ & 0.005 \\
\hline & & $\operatorname{Lag} 5$ & $1.02(0.99-1.05)$ & 0.15 & $1.0002(0.97-1.03)$ & 0.55 & $1.01(1.005-1.02)$ & 0.005 \\
\hline & & $\operatorname{Lag} 6$ & $1.03(1.006-1.06)$ & 0.01 & $1.02(0.99-1.05)$ & 0.59 & $1.01(1.005-1.02)$ & 0.005 \\
\hline & & $\operatorname{Lag} 7$ & $1.04(1.02-1.07)$ & 0.001 & $1.03(1.004-1.06)$ & 0.024 & $1.01(1.005-1.02)$ & 0.005 \\
\hline & $\mathrm{PM}_{10}$ & Lag 0 & $1.01(1.001-1.02)$ & 0.03 & $1.015(1.005-1.024)$ & 0.009 & $1.01(1.005-1.02)$ & 0.01 \\
\hline & & Lag 1 & $0.999(0.99-1.008)$ & 0.88 & $0.995(0.984-1.007)$ & 0.45 & $0.995(0.99-1.002)$ & 0.27 \\
\hline & & $\operatorname{Lag} 2$ & 0.996(0.99-1.005) & 0.40 & $0.996(0.985-1.008)$ & 0.64 & $0.995(0.99-1.002)$ & 0.27 \\
\hline
\end{tabular}


Table 4 RRs (95\% Cls) of respiratory admissions with an increase of $10 \mu \mathrm{g} / \mathrm{m}^{3}$ in air pollutants (and $1 \mathrm{mg} / \mathrm{m}^{3}$ in CO) according to single lag, adjusted unconstrained and constrained DLM models for each air pollutant among two age groups (Continued)

\begin{tabular}{|c|c|c|c|c|c|c|c|c|}
\hline & & $\operatorname{Lag} 3$ & 0.998(0.99-1.007) & 0.70 & $1.005(0.99-1.02)$ & 0.45 & $1.0003(0.997-1.003)$ & 0.94 \\
\hline & & $\operatorname{Lag} 4$ & $0.99(0.98-1.002)$ & 0.12 & $0.99(0.98-1.002)$ & 0.12 & $1.0003(0.997-1.003)$ & 0.94 \\
\hline & & $\operatorname{Lag} 5$ & 0.996(0.99-1.004) & 0.31 & $0.993(0.98-1.007)$ & 0.58 & $1.0003(0.997-1.003)$ & 0.94 \\
\hline & & $\operatorname{Lag} 6$ & $1.006(0.997-1.014)$ & 0.16 & $1.007(0.996-1.02)$ & 0.38 & $1.0003(0.997-1.003)$ & 0.94 \\
\hline & & $\operatorname{Lag} 7$ & $1.006(0.998-1.014)$ & 0.13 & $1.005(0.995-1.015)$ & 0.48 & $1.0003(0.997-1.003)$ & 0.94 \\
\hline \multirow[t]{39}{*}{ Under60 } & $\mathrm{SO}_{2}$ & $\operatorname{Lag} 0$ & $1.007(0.997-1.017)$ & 0.16 & $1.007(0.996-1.02)$ & 0.25 & $1.008(0.997-1.02)$ & 0.21 \\
\hline & & Lag 1 & $1.002(0.99-1.012)$ & 0.74 & $1.0005(0.988-1.013)$ & 0.95 & $1.001(0.99-1.01)$ & 0.93 \\
\hline & & $\operatorname{Lag} 2$ & $1.002(0.99-1.01)$ & 0.64 & $1.001(0.988-1.014)$ & 0.80 & $1.001(0.99-1.01)$ & 0.93 \\
\hline & & $\operatorname{Lag} 3$ & $1.004(0.994-1.014)$ & 0.45 & $1.004(0.99-1.02)$ & 0.68 & 0.999(0.995-1.004) & 0.97 \\
\hline & & $\operatorname{Lag} 4$ & $1.001(0.99-1.01)$ & 0.82 & $1.003(0.99-1.015)$ & 0.45 & $0.999(0.995-1.004)$ & 0.97 \\
\hline & & $\operatorname{Lag} 5$ & $0.99(0.98-1.003)$ & 0.18 & 0.993(0.98-1.007) & 0.35 & 0.999(0.995-1.004) & 0.97 \\
\hline & & $\operatorname{Lag} 6$ & 0.993(0.98-1.005) & 0.25 & 0.993(0.98-1.007) & 0.41 & 0.999(0.995-1.004) & 0.97 \\
\hline & & $\operatorname{Lag} 7$ & $1.003(0.99-1.014)$ & 0.57 & $1.01(0.996-1.023)$ & 0.22 & $0.999(0.995-1.004)$ & 0.97 \\
\hline & $\mathrm{CO}$ & $\operatorname{Lag} 0$ & $1.05(1.001-1.10)$ & 0.04 & $1.08(1.02-1.14)$ & 0.044 & $1.07(1.02-1.03)$ & 0.036 \\
\hline & & $\operatorname{Lag} 1$ & $0.98(0.93-1.03)$ & 0.40 & $0.97(0.91-1.03)$ & 0.14 & $0.97(0.94-1.002)$ & 0.075 \\
\hline & & $\operatorname{Lag} 2$ & $0.95(0.90-1.0002)$ & 0.051 & $0.96(0.90-1.02)$ & 0.50 & $0.97(0.94-1.002)$ & 0.075 \\
\hline & & $\operatorname{Lag} 3$ & $0.96(0.91-1.01)$ & 0.10 & $0.999(0.94-1.06)$ & 0.88 & $0.986(0.97-1.002)$ & 0.11 \\
\hline & & $\operatorname{Lag} 4$ & $0.94(0.89-0.99)$ & 0.02 & $0.934(0.88-0.99)$ & 0.03 & $0.986(0.97-1.002)$ & 0.11 \\
\hline & & $\operatorname{Lag} 5$ & $1.003(0.95-1.05)$ & 0.89 & $1.06(0.998-1.12)$ & 0.054 & $0.986(0.97-1.002)$ & 0.11 \\
\hline & & $\operatorname{Lag} 6$ & $0.95(0.90-1.001)$ & 0.051 & $0.96(0.90-1.02)$ & 0.12 & $0.986(0.97-1.002)$ & 0.11 \\
\hline & & $\operatorname{Lag} 7$ & $0.96(0.91-1.01)$ & 0.10 & $0.98(0.92-1.04)$ & 0.54 & $0.986(0.97-1.002)$ & 0.11 \\
\hline & $\mathrm{NO}_{2}$ & $\operatorname{Lag} 0$ & $1.02(0.99-1.05)$ & 0.17 & 1.02(0.98-1.06) & 0.22 & $1.03(1.0004-1.06)$ & 0.04 \\
\hline & & $\operatorname{Lag} 1$ & $1.006(0.98-1.03)$ & 0.63 & $1.01(0.97-1.05)$ & 0.61 & $0.98(0.96-1.002)$ & 0.18 \\
\hline & & $\operatorname{Lag} 2$ & $0.98(0.95-1.01)$ & 0.14 & $0.96(0.92-1.002)$ & 0.09 & $0.98(0.96-1.002)$ & 0.18 \\
\hline & & Lag 3 & $0.99(0.96-1.02)$ & 0.48 & $0.99(0.95-1.03)$ & 0.86 & $1.004(0.996-1.012)$ & 0.40 \\
\hline & & $\operatorname{Lag} 4$ & $1.01(0.98-1.04)$ & 0.56 & $1.002(0.96-1.04)$ & 0.97 & $1.004(0.996-1.012)$ & 0.40 \\
\hline & & $\operatorname{Lag} 5$ & $1.024(0.997-1.05)$ & 0.08 & $1.03(0.99-1.07)$ & 0.33 & $1.004(0.996-1.012)$ & 0.40 \\
\hline & & $\operatorname{Lag} 6$ & $1.01(0.98-1.04)$ & 0.35 & $1.033(0.99-1.076)$ & 0.23 & $1.004(0.996-1.012)$ & 0.40 \\
\hline & & $\operatorname{Lag} 7$ & $0.983(0.956-1.009)$ & 0.20 & $0.96(0.93-0.995)$ & 0.045 & $1.004(0.996-1.012)$ & 0.40 \\
\hline & $\mathrm{O}_{3}$ & $\operatorname{Lag} 0$ & 0.999(0.988-1.01) & 0.99 & $0.98(0.965-0.997)$ & 0.035 & $0.985(0.97-1.001)$ & 0.09 \\
\hline & & $\operatorname{Lag} 1$ & $1.02(1.01-1.03)$ & 0.01 & $1.03(1.01-1.05)$ & 0.004 & $1.01(1.003-1.02)$ & 0.01 \\
\hline & & $\operatorname{Lag} 2$ & $1.01(0.999-1.02)$ & 0.07 & $0.997(0.978-1.016)$ & 0.52 & $1.01(1.003-1.02)$ & 0.01 \\
\hline & & $\operatorname{Lag} 3$ & $1.01(0.999-1.02)$ & 0.054 & $1.01(0.99-1.03)$ & 0.47 & $0.999(0.996-1.003)$ & 0.50 \\
\hline & & $\operatorname{Lag} 4$ & $1.006(0.994-1.018)$ & 0.29 & 0.998(0.98-1.018) & 0.59 & $0.999(0.996-1.003)$ & 0.50 \\
\hline & & $\operatorname{Lag} 5$ & $1.005(0.993-1.017)$ & 0.40 & $1.003(0.98-1.02)$ & 0.82 & $0.999(0.996-1.003)$ & 0.50 \\
\hline & & $\operatorname{Lag} 6$ & $1.004(0.992-1.016)$ & 0.50 & $1.002(0.98-1.02)$ & 0.53 & $0.999(0.996-1.003)$ & 0.50 \\
\hline & & $\operatorname{Lag} 7$ & $1.001(0.99-1.01)$ & 0.86 & 0.993(0.976-1.01) & 0.26 & $0.999(0.996-1.003)$ & 0.50 \\
\hline & $\mathrm{PM}_{2.5}$ & $\operatorname{Lag} 0$ & $0.997(0.98-1.02)$ & 0.75 & $0.999(0.98-1.02)$ & 0.84 & $1.001(0.98-1.02)$ & 0.93 \\
\hline & & $\operatorname{Lag} 1$ & $0.996(0.98-1.02)$ & 0.68 & $0.999(0.98-1.02)$ & 0.99 & $0.99(0.98-1.01)$ & 0.28 \\
\hline & & $\operatorname{Lag} 2$ & $0.99(0.97-1.006)$ & 0.16 & $0.99(0.97-1.01)$ & 0.25 & $0.99(0.98-1.01)$ & 0.28 \\
\hline & & $\operatorname{Lag} 3$ & $0.994(0.97-1.01)$ & 0.59 & $1.003(0.98-1.03)$ & 0.93 & $1.003(0.996-1.01)$ & 0.33 \\
\hline & & $\operatorname{Lag} 4$ & $0.997(0.98-1.02)$ & 0.81 & $0.999(0.97-1.02)$ & 0.69 & $1.003(0.996-1.01)$ & 0.33 \\
\hline & & $\operatorname{Lag} 5$ & $1.002(0.98-1.022)$ & 0.84 & $1.006(0.98-1.03)$ & 0.31 & $1.003(0.996-1.01)$ & 0.33 \\
\hline & & $\operatorname{Lag} 6$ & $0.999(0.98-1.02)$ & 0.91 & $0.989(0.96-1.01)$ & 0.25 & $1.003(0.996-1.01)$ & 0.33 \\
\hline
\end{tabular}


Table 4 RRs (95\% Cls) of respiratory admissions with an increase of $10 \mu \mathrm{g} / \mathrm{m}^{3}$ in air pollutants (and $1 \mathrm{mg} / \mathrm{m}^{3}$ in CO) according to single lag, adjusted unconstrained and constrained DLM models for each air pollutant among two age groups (Continued)

\begin{tabular}{rlllllll}
\hline & Lag 7 & $1.02(0.997-1.04)$ & 0.10 & $1.02(0.997-1.04)$ & 0.22 & $1.003(0.996-1.01)$ & 0.33 \\
$P_{10} 10$ & $\operatorname{Lag} 0$ & $1.002(0.99-1.01)$ & 0.51 & $1.008(1.0004-1.015)$ & 0.025 & $1.01(0.999-1.015)$ & 0.17 \\
& $\operatorname{Lag} 1$ & $0.99(0.98-0.999)$ & 0.04 & $0.988(0.98-0.997)$ & 0.01 & $0.994(0.99-0.998)$ & 0.008 \\
& $\operatorname{Lag} 2$ & $0.99(0.987-1.001)$ & 0.12 & $0.999(0.99-1.01)$ & 0.95 & $0.994(0.99-0.998)$ & 0.008 \\
& $\operatorname{Lag} 3$ & $0.997(0.99-1.003)$ & 0.31 & $0.996(0.99-1.005)$ & 0.38 & $1.0001(0.998-1.002)$ & 0.45 \\
& $\operatorname{Lag} 4$ & $0.998(0.99-1.005)$ & 0.60 & $0.999(0.99-1.008)$ & 0.85 & $1.0001(0.998-1.002)$ & 0.45 \\
& $\operatorname{Lag} 5$ & $1.001(0.99-1.01)$ & 0.75 & $1.002(0.993-1.01)$ & 0.30 & $1.0001(0.998-1.002)$ & 0.45 \\
& $\operatorname{Lag} 6$ & $1.002(0.995-1.01)$ & 0.62 & $1.001(0.992-1.01)$ & 0.61 & $1.0001(0.998-1.002)$ & 0.45 \\
& $\operatorname{Lag} 7$ & $1.002(0.996-1.01)$ & 0.49 & $0.999(0.99-1.008)$ & 0.96 & $1.0001(0.998-1.002)$ & 0.45 \\
\hline
\end{tabular}

hospitalizations [22]. Another study in Tehran, Iran found that total respiratory diseases hospitalizations were increased by $1 \%$ for each $10 \mu \mathrm{g} / \mathrm{m}^{3}$ increase in $\mathrm{NO}_{2}$ level [23]. A study from Lanzhou, China also reported that total respiratory disease hospitalizations were increased by $11.0 \%$ for each $10 \mu \mathrm{g} / \mathrm{m}^{3}$ increase of $\mathrm{NO}_{2}$ on dust days and by $2.5 \%$ for $10 \mu \mathrm{g} / \mathrm{m}^{3}$ increases in $\mathrm{NO}_{2}$ on non-dust days [21]. Tao et al in Lanzhou, China, found that total respiratory disease hospitalizations increased by $1.1 \%$ for each $10 \mu \mathrm{g} / \mathrm{m}^{3}$ increase in $\mathrm{NO}_{2}$ levels [20]. In contrast, a previous study from Europe found no significant associations between $\mathrm{NO}_{2}$ and respiratory diseases hospitalizations. In the mentioned study, the mean daily concentrations of $\mathrm{NO}_{2}$ was $50.3 \mathrm{ppb}$ [54]. Another study done by Rezaei et al in 2016, did not find a significant association between $\mathrm{NO}_{2}$ concentrations and respiratory disease hospitalizations in Kerman, Iran. The mean annual concentrations of $\mathrm{NO}_{2}$ was very low and equal to $0.04 \mathrm{ppm}$ in the Kerman study [55], which is lower than the present study that the mean annual concentrations of $\mathrm{NO}_{2}$ was $53.45 \mu \mathrm{g} / \mathrm{m}^{3}$.

Several studies investigated the effects of air pollution on human health, in single-pollutant models, among different gender and age groups [13, 15, 21, 36, 49]. In this study, there were different health effects of air pollution between males and females in regard to respiratory admissions. Some studies observed different health effects of air pollution between two genders. In the

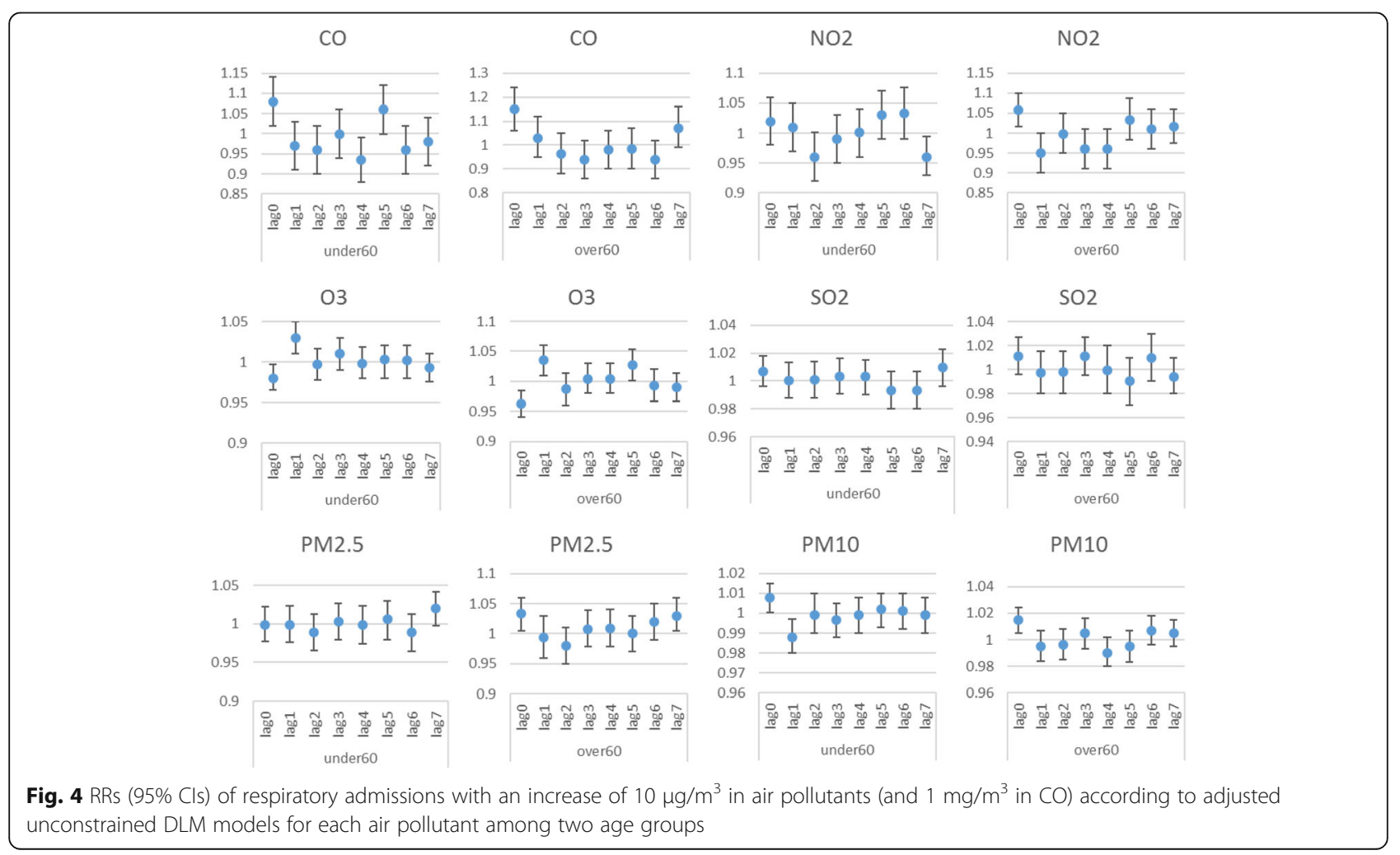




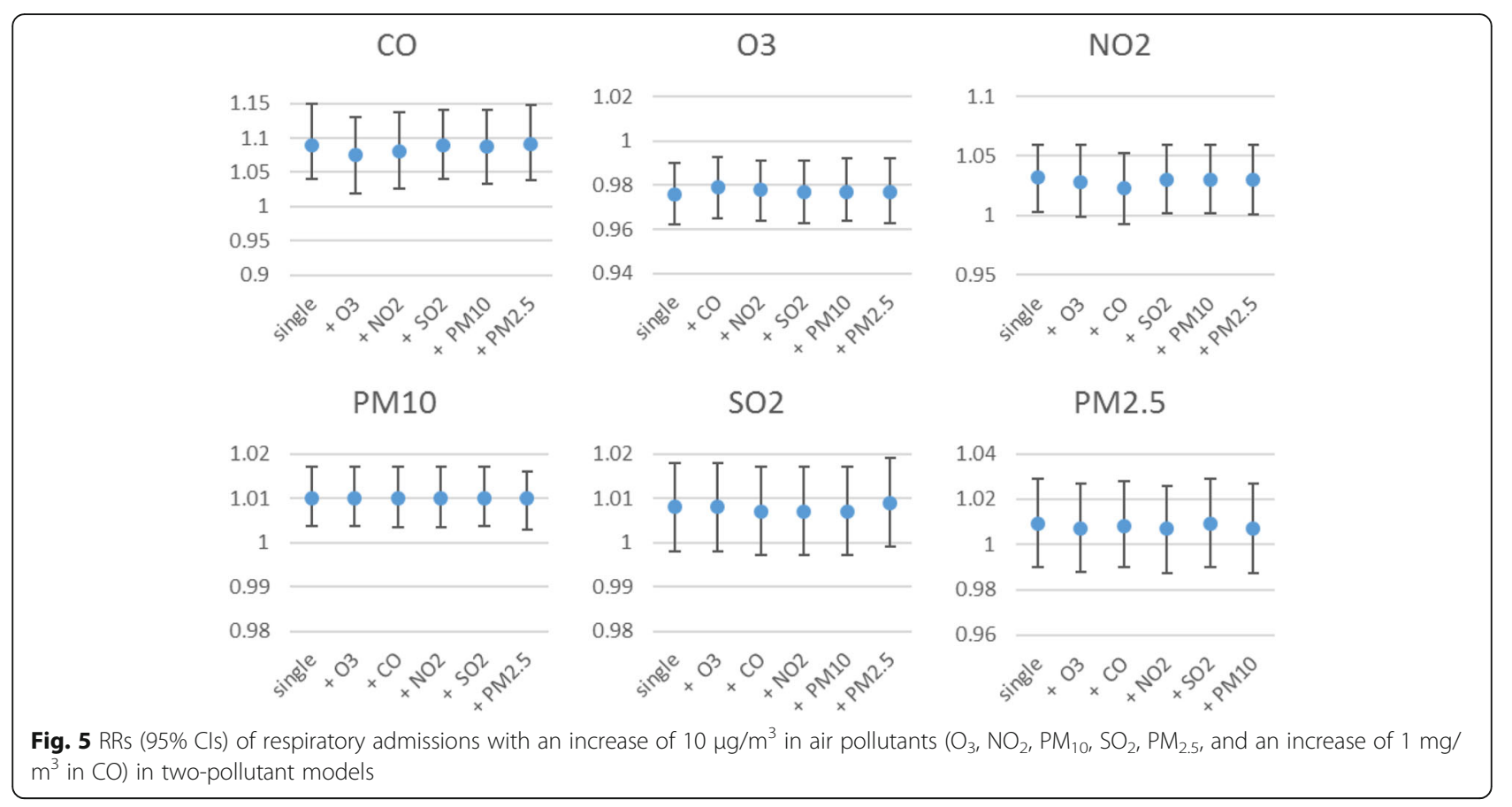

current study, significant adverse effects were observed for $\mathrm{CO}$ at lag 0 and lag7 day and $\mathrm{PM}_{2.5}$ at lag-7 in females, while a higher risk of respiratory hospital admissions was seen in males for $\mathrm{PM}_{10}$ at lag $0, \mathrm{CO}$ at lag 0 , $\mathrm{SO}_{2}$ at lag $0, \mathrm{NO}_{2}$ at lag 0 and lag 6 day, and $\mathrm{O}_{3}$ at lag 1 days. These results could be related to men's occupation in jobs such as industry or taxi driving or more outdoor activities which expose them to more air pollution[56, 57]. This study also found that older adults (60+ year-olds) were more vulnerable to respiratory disease exacerbations. These findings are consistent with previous studies that the elderly were more susceptible to exposure to outdoor air pollution [1, 20, 21].

One of the limitations of this study was missing air pollutant data. Missing data is a frequent problem in many scientific fields, especially in studies about the effects of ambient air pollutants $[34,58]$. Missing data is common in air quality monitoring stations due to unpredicted technical malfunctions or faulty equipment, that effect data storage [34]. There are three types of missing data according to their generation, including missing completely at random (MCAR), missing at random (MAR) and missing not at random (MNAR) [59, 60]. In the present study missing data occurred due to failure of air quality monitoring stations and it was not related to the pollutant levels in certain days.

There are many methods for dealing with missing data. For example, complete case analysis is the default method used by most statistical software that exclude incomplete observations from analysis. But under the MCAR and MAR assumption, if a high proportion of incomplete observations are excluded, loss of precision may happen
[34]. Also in time series analysis, excluding this observations by using the complete case method may impair the temporal pattern of the data, including trends, seasonality and autocorrelation $[34,61]$. In this study, the missing data imputed by using the expectation-maximization (EM) algorithm method [34].

However, additional analysis was also done by using complete case analysis. Result of complete case analysis generally showed the same results but with lower precision compared to EM imputed data. However, the effect of $\mathrm{SO}_{2}$ at lag $0(\mathrm{RR}, 95 \% \mathrm{CI}=1.02,(1.006-1.035))$ and $\mathrm{PM}_{2.5}$ at lag $0(\mathrm{RR}, 95 \% \mathrm{CI}=1.03$, (1.006-1.055)) was significant in complete case analysis, but not in EM algorithm imputed data. These findings further emphasize the negative effects of air pollutants in Arak.

The other limitations of this study were that in ecological studies, such as the current study, the results cannot be directly inferred to individual levels. Another limitation of this study was limiting the cases to two major hospitals in Arak. Also, we were not able to control potential individual confounders, such as smoking, genetic susceptibility or migrations, and population displacements.

\section{Conclusion}

The results of this investigation show that some outdoor air pollutants were associated with increased respiratory hospital admissions. The strongest association was seen for $\mathrm{CO}$ and $\mathrm{NO}_{2}$. This study also found evidence that males and elderly age groups are more susceptible to air pollutants. These findings suggest new evidence about the health effects of air pollutions, in the Middle East 
region, which despite its increased motor vehicles, traffic and industrialization has not yet adopted appropriate strategies to control air pollution.

\section{Abbreviations}

Cl: Confidence interval; CO: Carbon monoxide; Df: Degrees of freedom; DLM: Distributed Lag Models; DOW: Day of the week; ER: Emergency room; GLM: Generalized linear models; ICD: International classification of diseases; IQR: Interquartile range; $\mathrm{NO}_{2}$ : Nitrogen dioxide; $\mathrm{O}_{3}$ : Ozone; $\mathrm{PM}_{10}$ : Particulate matter 10 micrometers or less in diameter; $\mathrm{PM}_{2.5}$ : Particulate matter 2.5 micrometers or less in diameter; Ppb: Parts per billion; RR: Rate Ratio; $\mathrm{SO}_{2}$ : Sulfur dioxide

\section{Acknowledgements}

This research was financially supported by the Kerman University of Medical Sciences. The authors are grateful to Kerman University of Medical Sciences, Arak University of Medical Sciences, and the Arak Department of Environment and Meteorological Organization for their cooperation in this study.

\section{Funding}

This research was financially supported by the Kerman University of Medical Sciences (Grant No 95-248).

\section{Availability of data and materials}

Please contact author for data requests.

\section{Authors' contributions}

MV: Wrote the initial proposal, inquired the data, cleaned the data, did the statistical analysis, wrote the initial draft. NK: Suggested the topic, commented on the proposal and methods, provided scientific support, supervised the research and data analysis, edited and commented on the final draft. MM: Helped in cleaning the data, cooperated in the statistical analysis, commented on the final draft. AK: commented on the proposal and methods, provided scientific support, edited and commented on the final draft. All authors read and approved the final manuscript

\section{Competing interest}

The authors declare that they have no competing interests.

\section{Consent for publication}

Not applicable.

\section{Ethics approval and consent to participate}

The study protocol was approved by the Institutional Review Board of the Faculty of Health, Kerman University of Medical Sciences and was also reviewed and approved by the Standing Committee on Ethics in Research of both the Arak (Ethical Code: IR.ARAKMU.REC.1395.80) and Kerman University of Medical Sciences (Ethical Code: IR.KMU.REC.1395.248). Written informed consent from individuals was not required because we used aggregated and anonymous data.

\section{Publisher's Note}

Springer Nature remains neutral with regard to jurisdictional claims in published maps and institutional affiliations.

\section{Author details}

'Neurology Research Center, Kerman University of Medical Sciences, Kerman, Iran. ${ }^{2}$ Environmental Health Engineering Research Center, Kerman University of Medical Sciences, Kerman, Iran. ${ }^{3}$ Modeling in Health Research Center, Institute for Futures Studies in Health, Kerman University of Medical Sciences, Kerman, Iran. ${ }^{4}$ Department of Environmental Health Engineering, Faculty of Health, Arak University of Medical Sciences, Arak, Iran. ${ }^{5}$ Department of Epidemiology and Biostatistics, School of Public Health, Kerman University of Medical Sciences, Kerman, Iran.
Received: 8 February 2017 Accepted: 14 June 2017

Published online: 17 July 2017

\section{References}

1. Zhang Y, Wang SG, Ma YX, Shang KZ, Cheng YF, Li X, et al. Association between ambient Air pollution and hospital emergency admissions for respiratory and cardiovascular diseases in Beijing: a time series study. Biomed Environ Sci. 2015;28(5):352-63.

2. Lai HK, Tsang H, Wong CM. Meta-analysis of adverse health effects due to air pollution in Chinese populations. BMC Public Health. 2013;13:360. Pubmed Central PMCID: PMC3698155, Epub 2013/04/19. eng.

3. Daryanoosh SM, Goudarzi G, Harbizadeh A, Nourmoradi H, Vaisi AA, Armin H. Hospital admission for respiratory and cardiovascular diseases Due to particulate matter in ilam, Iran. Jundishapur J Health Sci. 2016;9:1.

4. Mills NL, Donaldson K, Hadoke PW, Boon NA, MacNee W, Cassee FR, et al. Adverse cardiovascular effects of air pollution. Nat Clin Pract Cardiovasc Med. 2009;6(1):36-44.

5. WHO. Fact Sheet on Outdoor Air Pollution (N"133): Ambient Air Quality and Health, Updated March 2014. [Available online: http://www.who.int/ mediacentre/factssheets/fs313/en/(accessed on 1 Mar 2014).].

6. Fung K, Luginaah I, Gorey K, Webster G. Air pollution and daily hospitalization rates for cardiovascular and respiratory diseases in London, Ontario. Int J Environ Stud. 2005;62(6):677-85.

7. Zemp E, Elsasser S, Schindler C, Kunzli N, Perruchoud AP, Domenighetti G, et al. Long-term ambient air pollution and respiratory symptoms in adults (SAPALDIA study). Am J Respir Crit Care Med. 1999:159(4):1257-66.

8. Dominici F, McDermott A, Daniels M, Zeger SL, Samet JM. Revised analyses of the national morbidity, mortality, and air pollution study: mortality among residents of 90 cities. J Toxicol Environ Health Part A. 2005;68(13-14):1071-92.

9. Carugno M, Consonni D, Randi G, Catelan D, Grisotto L, Bertazzi PA, et al. Air pollution exposure, cause-specific deaths and hospitalizations in a highly polluted Italian region. Environ Res. 2016;147:415-24.

10. Dadbakhsh M, Khanjani N, Bahrampour A. Death from respiratory diseases and Air pollutants in Shiraz, Iran (2006-2012). J Environ Pollution Human Health. 2015:3(1):4-11, doi:10.12691/jephh-3-1-2

11. Dastoorpoor M, Idani E, Khanjani N, Goudarzi G, Bahrampour A. Relationship between Air pollution, weather, traffic, and traffic-related mortality. Trauma Monthly. 2016;21(4):e37585

12. Hashemi SY, Khanjani N. Air pollution and cardiovascular hospital admissions in Kerman, Iran. J Heart Cardiology. 2016;2(2):1-6.

13. Rodopoulou S, Chalbot M-C, Samoli E, DuBois DW, San Filippo BD, Kavouras IG. Air pollution and hospital emergency room and admissions for cardiovascular and respiratory diseases in doña Ana county, New Mexico. Environ Res. 2014:129:39-46.

14. Anderson H, Spix C, Medina S, Schouten J, Castellsague J, Rossi G, et al. Air pollution and daily admissions for chronic obstructive pulmonary disease in 6 European cities: results from the APHEA project. Eur Respir J. 1997;10(5): 1064-71.

15. Tramuto F, Cusimano R, Cerame G, Vultaggio M, Calamusa G, Maida CM, et al. Urban air pollution and emergency room admissions for respiratory symptoms: a case-crossover study in Palermo, Italy. Environ Health. 2011 10(1):1.

16. Zanobetti A, Schwartz J, Dockery DW. Airborne particles are a risk factor for hospital admissions for heart and lung disease. Environ Health Perspect. 2000:108(11):1071

17. Zanobetti A, Franklin M, Koutrakis P, Schwartz J. Fine particulate air pollution and its components in association with cause-specific emergency admissions. Environ Health. 2009;8(1):1.

18. Schwartz J, Spix C, Touloumi G, Bachárová L, Barumamdzadeh T, le Tertre A et al. Methodological issues in studies of air pollution and daily counts of deaths or hospital admissions. J Epidemiol Community Health. 1996:50 Suppl 1:S3-S11.

19. Wilson AM, Wake CP, Kelly T, Salloway JC. Air pollution, weather, and respiratory emergency room visits in two northern New England cities: an ecological time-series study. Environ Res. 2005;97(3):312-21.

20. Tao Y, Mi S, Zhou S, Wang S, Xie X. Air pollution and hospital admissions for respiratory diseases in Lanzhou. China Environ Pollut. 2014;185:196-201.

21. Ma Y, Xiao B, Liu C, Zhao Y, Zheng X. Association between ambient Air pollution and emergency room visits for respiratory diseases in spring dust storm season in Lanzhou, china. Int J Environ Res Public Health. 2016:13(6): 613. 
22. Liu P, Wang X, Fan J, Xiao W, Wang Y. Effects of Air pollution on hospital emergency room visits for respiratory diseases: urban-suburban differences in Eastern China. Int J Environ Res Public Health. 2016;13(3):341.

23. Shahi AM, Omraninava A, Goli M, Soheilarezoomand HR, Mirzaei N. The effects of air pollution on cardiovascular and respiratory causes of emergency admission. Emergency. 2014;2(3):107.

24. Wong TW, Lau TS, Yu TS, Neller A, Wong SL, Tam W, et al. Air pollution and hospital admissions for respiratory and cardiovascular diseases in Hong Kong. Occup Environ Med. 1999;56(10):679-83.

25. Su C, Breitner S, Schneider A, Liu L, Franck U, Peters A, et al. Short-term effects of fine particulate air pollution on cardiovascular hospital emergency room visits: A time-series study in Beijing, China. Int Arch Occup Environ Health. 2016;89(4):641-57.

26. Berend N. Contribution of air pollution to COPD and small airway dysfunction. Respirology. 2016;21(2):237-44.

27. Hashemi SY, Khanjani N, Soltaninejad Y, Momenzadeh R. Air pollution and cardiovascular mortality in Kerman from 2006 to 2011. Am J Cardiovasc Dis Res. 2014;2(2):27-30.

28. Poursafa P, Kelishadi R, Ghasemian A, Sharifi F, Djalalinia S, Khajavi A, et al. Trends in health burden of ambient particulate matter pollution in Iran, 1990-2010: findings from the global burden of disease study 2010. Environ Sci Pollut Res. 2015;22(23):18645-53.

29. Dadbakhsh M, Khanjani N, Bahrampour A. Death from cardiovascular diseases and Air pollution in Shiraz, Iran (march 2006-march 2012). J Epidemiol Prev Med. 2016;2(1):114.

30. Fazelinia F, Khodabandehlou AA, Rafati L, Mahvi AH. Investigation of air quality index and PM10 and PM2. 5 in Arak. Iranian J Health Sci. 2013;1(3):12-7.

31. Solgi E. Assessment of Copper and Zinc Contamination in Soils of Industrial Estates of Arak Region (Iran). Iranian J Toxicology Volume. 2015;9(28):1277-83.

32. Mirhossaini S, Pourzamani H. Evaluation and analysis of the environmental noise of Arak, Iran. J Appl Sci. 2008;8(7):1333-6.

33. Hosseini V, Shahbazi H. Urban Air pollution in Iran. Iran Stud. 2016:49(6):1029-46.

34. Junger $W$, de Leon AP. Imputation of missing data in time series for air pollutants. Atmos Environ. 2015;102:96-104.

35. Bhaskaran K, Gasparrini A, Hajat S, Smeeth L, Armstrong B. Time series regression studies in environmental epidemiology. Int J Epidemiol. 2013

36. Phung D, Hien TT, Linh HN, Luong LM, Morawska L, Chu C, et al. Air pollution and risk of respiratory and cardiovascular hospitalizations in the most populous city in Vietnam. Sci Total Environ. 2016;557:322-30.

37. Ye X, Peng L, Kan H, Wang W, Geng F, Mu Z, et al. Acute effects of particulate Air pollution on the incidence of coronary heart disease in Shanghai. China PloS ONE. 2016:11(3):e0151119.

38. http://cran.r-project.org/bin/windows/base/. Accessed 21 June 2016

39. Gasparrini A. Distributed lag linear and non-linear models in $\mathrm{R}$ : the package dlnm. J Stat Softw. 2011;43(8):1

40. World Health Organization: 2014 Air Pollution Ranking.[Posted on May 16th 2015]. Available at: http:faq/2015-05-16/world-health-organization-2014-airpollution-ranking/.Accessed 29 Nov 2016.

41. Yang T-H, Masumi S-I, Weng S-P, Chen H-W, Chuang H-C, Chuang K-J. Personal exposure to particulate matter and inflammation among patients with periodontal disease. Sci Total Environ. 2015;502:585-9.

42. Atkinson RW, Ross Anderson H, Sunyer J, Ayres J, Baccini M, Vonk JM, et al. Acute effects of particulate air pollution on respiratory admissions: results from APHEA 2 project. Am J Respir Crit Care Med. 2001;164(10):1860-6.

43. Yi O, Hong $\mathrm{Y}$-C, Kim H. Seasonal effect of PM10 concentrations on mortality and morbidity in Seoul, Korea: A temperature-matched case-crossover analysis. Enviro Res. 2010;110(1):89-95.

44. Medina-Ramón M, Zanobetti A, Schwartz J. The effect of ozone and PM10 on hospital admissions for pneumonia and chronic obstructive pulmonary disease: a national multicity study. Am J Epidemiol. 2006;163(6):579-88.

45. Ji M, Cohan DS, Bell ML. Meta-analysis of the association between shortterm exposure to ambient ozone and respiratory hospital admissions. Environ Res Lett. 2011;6(2), 024006.

46. Bell ML, Zanobetti A, Dominici F. Who is more affected by ozone pollution? a systematic review and meta-analysis. Am J Epidemiol. 2014:180(1):15-28.

47. Slaughter JC, Kim E, Sheppard L, Sullivan JH, Larson TV, Claiborn C. Association between particulate matter and emergency room visits, hospital admissions and mortality in Spokane, Washington. J Expo Sci Environ Epidemiol. 2005;15(2):153-9.
48. Xu Q, Li X, Wang S, Wang C, Huang F, Gao Q, et al. Fine particulate Air pollution and hospital emergency room visits for respiratory disease in urban areas in Beijing, China, in 2013. PLoS ONE. 2016;11(4):e0153099.

49. Samoli E, Atkinson RW, Analitis A, Fuller GW, Green DC, Mudway I, et al. Associations of short-term exposure to traffic-related air pollution with cardiovascular and respiratory hospital admissions in London, UK. Occupational and environmental medicine. 2016:oemed-2015-103136.

50. Anderson H, Atkinson R, Bremner S, Carrington J, Peacock J. Quantitative systematic review of short term associations between ambient air pollution (particulate matter, ozone, nitrogen dioxide, sulphur dioxide and carbon monoxide), and mortality and morbidity. Report to Department of Health revised following first review. 2007 June.

51. Chen R, Chu C, Tan J, Cao J, Song W, Xu X, et al. Ambient air pollution and hospital admission in Shanghai, China. J Hazard Mater. 2010;181(1):234-40.

52. Zhen WM, Shan Z, Gong WS, Yan T, Zheng SK. The weather temperature and air pollution interaction and its effect on hospital admissions due to respiratory system diseases in western China. Biomed Environ Sci. 2013: 26(5):403-7.

53. Kan H, London SJ, Chen G, Zhang Y, Song G, Zhao N, et al. Season, sex, age, and education as modifiers of the effects of outdoor air pollution on daily mortality in Shanghai, China: The Public Health and Air Pollution in Asia (PAPA) Study. Environ Health Perspect. 2008;116(9):1183-8. Pubmed Central PMCID: PMC2535620, Epub 2008/09/17. eng

54. Atkinson RW, Bremner SA, Anderson HR, Strachan DP, Bland JM, de Leon AP. Short-term associations between emergency hospital admissions for respiratory and cardiovascular disease and outdoor air pollution in London. Arch Environ Health. 1999;54(6):398-411. Epub 2000/01/14. eng.

55. Rezaei S, Khanjani N, Mohammadi Senjedkooh S, Darabi FZ. The effect of Air pollution on respiratory disease visits to the emergency department in Kerman, Iran. J Health Dev. 2016;4(4):306-14.

56. Khanjani N, Ranadeh Kalankesh L, Mansouri F. Air pollution and respiratory deaths in Kerman, Iran (from 2006 till 2010). Iranian J Epidemiol. 2012;8(3): $58-65$.

57. Wu S, Deng F, Niu J, Huang Q, Liu Y, Guo X. Association of heart rate variability in taxi drivers with marked changes in particulate air pollution in Beijing in. Environ Health Perspect. 2008;2010:87-91.

58. Moshenberg S, Lerner U, Fishbain B. Spectral methods for imputation of missing air quality data. Environ Syst Res. 2015;4(1):26.

59. Kang $\mathrm{H}$. The prevention and handling of the missing data. Korean J Anesthesiol. 2013;64(5):402-6.

60. Rubin DB. Inference and missing data. Biometrika. 1976;63(3):581-92.

61. Dempster AP, Laird NM, Rubin DB. Maximum likelihood from incomplete data via the EM algorithm. J Royal Statistical Society Series B (Methodological). 1977:39(1):1-38.

\section{Submit your next manuscript to BioMed Central and we will help you at every step:}

- We accept pre-submission inquiries

- Our selector tool helps you to find the most relevant journal

- We provide round the clock customer support

- Convenient online submission

- Thorough peer review

- Inclusion in PubMed and all major indexing services

- Maximum visibility for your research

Submit your manuscript at www.biomedcentral.com/submit 